\title{
THE ORIGIN OF THALAMIC INPUTS TO THE ARCUATE PREMOTOR AND SUPPLEMENTARY MOTOR AREAS ${ }^{1}$
}

\author{
GERALD R. SCHELL AND PETER L. STRICK ${ }^{2}$ \\ Research Service, Veterans Administration Medical Center, and Departments of Neurosurgery and Physiology, State University \\ of New York-Upstate Medical Center, Syracuse, New York, 13210
}

Received June 13, 1983; Revised August 8, 1983; Accepted August 16, 1983

\begin{abstract}
We have used retrograde transport of wheat germ agglutinin conjugated to horseradish peroxidase to examine the origin of thalamic input to the two premotor areas with the densest projections to the motor cortex: the arcuate premotor area (APA) and the supplementary motor area (SMA). Retrograde transport demonstrated that the two premotor areas and the motor cortex each receive thalamic input from separate, cytoarchitectonically well defined subdivisions of the ventrolateral thalamus. According to the nomenclature of Olszewski (Olszewski, J. (1952) The Thalamus of the Macaca mulatta. An Atlas for Use with the Stereotaxic Instrument, S. Karger, AG, Basel), input to the APA originates largely from area $\mathrm{X}$, input to the SMA originates largely from the pars oralis subdivision of nucleus ventralis lateralis (VLo), and that to the motor cortex originates largely from the pars oralis subdivision of nucleus ventralis posterior lateralis (VPLo). These observations, when combined with prior studies on the termination of various subcortical efferents in the thalamus, lead to the following scheme of connections: (1) rostral portions of the deep cerebellar nuclei project to the motor cortex via VPLo; (2) caudal portions of the deep cerebellar nuclei project to the APA via area X; and (3) the globus pallidus projects to the SMA via VLo. Thus, each thalamocortical pathway is associated with a distinct subcortical input.
\end{abstract}

The motor cortex has long been known to receive projections from regions in the frontal lobe which historically have been termed "premotor areas" (see Brinkman and Porter, 1979; Wiesendanger, 1981; Weinrich and Wise, 1982, for recent reviews). These premotor areas are thought to contribute to the programming of skilled movement and the sequencing of motor tasks. Despite the existence of concepts concerning premotor areas there is little information on their actual functions and anatomical connections.

Recent experiments by Muakkassa and Strick (1979) demonstrated that there are four spatially separate and somatotopically organized premotor areas which project directly to the motor cortex (see also Pandya and Kuypers, 1969; Jones and Powell, 1970; Pandya and Vignolo, 1971; Matsumura and Kubota, 1979). The densest pro-

\footnotetext{
${ }^{1}$ We thank Dr. Donna S. Hoffman and Ms. Laura Westfall for their critical review of this manuscript, Ms. Donna Kenyon and Ann Cochran for their expert technical assistance, and Dr. Robert B. King for his generous support. This project has been supported in part through funds from the Veterans Administration Medical Research Service, United States Public Health Service Grant 02957, and the Department of Neurosurgery, State University of New York.

${ }^{2}$ To whom correspondence should be addressed, at Research Service (151), V. A. Medical Center, Syracuse, NY 13210.
}

jections originate from two of the premotor areas. One of these is the arcuate premotor area (APA), ${ }^{3}$ located in and around the caudal bank of the arcuate sulcus; the other is the supplementary motor area (SMA), located on the medial wall of the hemisphere (e.g., Woolsey et al., 1952). A third premotor area was located in the ventral bank of the cingulate sulcus and a fourth in the cortex surrounding the inferior and superior precentral sulci. Based on the available anatomical and physiological evidence, a reasonable hypothesis is that these premotor areas represent elements in multiple parallel pathways which differentially control motor cortex output and motor behavior.

\footnotetext{
${ }^{3}$ The abbreviations used are: APA, arcuate premotor area; $\mathrm{CL}, \mathrm{Cl}$, nucleus centralis lateralis; $\mathrm{Cn}$ Md, nucelus centrum medianum; $\mathbf{G P i}$, globus pallidus internal segment; MD, nucleus medialis dorsalis; SMA, supplementary motor cortex; $\mathrm{SNpr}$, substantia nigra, pars reticulata; VAmc, nucleus ventralis anterior, pars magnocellularis; VApc, nucleus ventralis anterior, pars parvocellularis; VLc, nucleus ventralis lateralis, pars caudalis; VLm, nucleus ventralis lateralis, pars medialis; VLo, nucleus ventralis lateralis, pars oralis; VLps, nucleus ventralis lateralis, pars postrema; VPI, nucleus ventralis posterior inferior; VPLo, nucleus ventralis posterior lateralis, pars oralis; WGA-HRP, wheat germ agglutinin conjugated to horseradish peroxidase.
} 
We sought to understand how two major subcortical motor systems, the cerebellum and the basal ganglia, might differentially influence the premotor areas and motor cortex. Recent studies have shown that cerebellar, pallidal, and nigral efferents project to the ventrolateral thalamus and that there is little overlap in the sites of termination of these systems (e.g., Mehler, 1971; Kuo and Carpenter, 1973; Carpenter et al., 1976; Kim et al., 1976; Percheron, 1977; Stanton, 1980; Kalil, 1981; Devito and Anderson, 1982; Asanuma et al., 1983b). These studies have also indicated that, whereas cerebellar cfferents terminate in thalamic regions which project to motor cortex, efferents from the basal ganglia do not. However, the lack of precise information regarding the cortical projections of subdivisions of the ventrolateral thalamus has hampered attempts to link subcortical structures with particular cortical areas.

We have used retrograde transport of wheat germ agglutinin conjugated to horseradish peroxidase (WGA$\mathrm{HRP}$ ) to examine the origin of thalamic input to the two premotor areas with the densest projections to the motor cortex: the APA and the SMA. Retrograde transport demonstrated that the two premotor areas and the motor cortex each receive thalamic input from separate, cytoarchitectonically well defined subdivisions of the ventrolateral thalamus. According to the nomenclature of Olszewski (1952), input to the APA originates largely from area $\mathrm{X}$, input to the SMA originates largely from the pars oralis subdivision of nucleus ventralis lateralis (VLo), and that to the motor cortex originates largely from the pars oralis subdivision of nucleus ventralis posterior lateralis (VPLo). These observations, when combined with prior studies on the termination of various subcortical efferents in the thalamus, lead to the following scheme of connections: (1) rostral portions of the deep cerebellar nuclei project to the motor cortex via VPLo; (2) caudal portions of the deep cerebellar nuclei project to the APA via area X; and (3) the globus pallidus projects to the SMA via VLo. Thus, each thalamocortical pathway is associated with a distinct subcortical input.

\section{Materials and Methods}

This study is based on observations from seven monkeys (Macaca mulatta). Retrograde transport of WGAHRP (Sigma) was used to "label" the origin of thalamic input to the SMA, the APA, and parts of the motor cortex.

Each animal was anesthetized with ketamine hydrochloride and sodium pentobarbitol and mounted in a Kopf stereotaxic head holder. Under aseptic conditions a craniotomy was performed over the appropriate cortical area and the dura was opened. In five animals, we attempted to determine the full extent of the thalamic regions which project to the SMA, APA, and parts of the motor cortex (i.e., those regions adjacent to the central sulcus). To accomplish this, multiple injections of $2 \%$ WGA-HRP $(0.025$ to $0.05 \mu \mathrm{l})$ were placed unilaterally throughout one of the three cortical areas in separate animals. In another two monkeys, we attempted to determine the thalamic regions which projected to the "arm areas" of the SMA and APA by making more localized injections.

Various surface landmarks were used to guide the placement of all injection sites. The use of these landmarks was based on previous anatomical (Muakkassa and Strick, 1979; Matsumura and Kubota, 1979) and physiological (Woolsey et al., 1952; Brinkman and Porter, 1979b; Tanji and Kurata, 1982; Weinrich and Wise, 1982) studies. All injections were spaced $1 \mathrm{~mm}$ apart, except to avoid blood vessels, and were delivered through a 1- $\mu$ l Hamilton syringe with a 32 gauge needle. The injections into the APA and the motor cortex were made perpendicular to the cortical surface at a depth of 1 to $1.5 \mathrm{~mm}$ below the cortical surface and $1 \mathrm{~mm}$ from the relevant sulci. The SMA injections were placed between 2 and $4.5 \mathrm{~mm}$ below the surface and $1 \mathrm{~mm}$ from the medial edge of the hemisphere. In all cases, the needle was left in place for $3 \mathrm{~min}$ following the injections. During this procedure the cortex was kept moist with warm saline. At the end of the injection procedure, a Silastic sheet was placed over the remaining dura and glued in place. The wound was then closed and the animal was returned to a cage.

After a survival period of 3 days, each animal was reanesthetized and perfused transcardially using methods similar to those described by Rosene and Mesulam (1978). The perfusates included: (1) $0.1 \mathrm{M}$ phosphate buffer, pH 7.4 ; (2) $1 \%$ paraformaldehyde and $1.25 \%$ glutaraldehyde in $0.1 \mathrm{M}$ phosphate buffer; (3) a solution of the same aldehyde/buffer mixture with $10 \%$ glycerin added; and (4) a cold $0.1 \mathrm{M}$ phosphate buffer containing $10 \%$ glycerine. Following perfusion each brain was removed and placed in phosphate buffer containing $10 \%$ glycerine for $24 \mathrm{hr}$. A tissue block containing the cortical injection site and thalamus was sectioned serially in the frontal plane at $50 \mu \mathrm{m}$ on a freezing microtome. All sections were processed by the tetramethylbenzidine method according to Mesulam (1978) and Mesulam et al. (1980) with only minor modifications. Selected sections were counterstained with neutral red.

The material was studied microscopically with brightand darkfield illumination. Surface maps of the injection sites were reconstructed from enlarged drawings $(\times 10)$ of individual sections. Injection sites were considered to include the densely stained regions adjacent to each needle track, but not the lightly stained "halo" surrounding these regions. Labeled neurons in thalamic sections were examined at $\times 100$ or $\times 400$. The location of each labeled neuron was charted using an X-Y plotter system fed from linear potentiometers coupled to the two axes of movement in the microscope stage. At least every fourth thalamic section was plotted in this manner. After sections at selected levels had been plotted they were photographed ( $\times 10$ to $\times 25$ magnification). These sections were then counterstained with thionin and rephotographed. The cytoarchitecture of labeled areas was examined and thalamic borders were drawn using these counterstained sections and the microscope-coupled plotting system.

Serial sections of the thalamus from two normal rhesus monkeys (embedded in celloidin, cut at $50 \mu \mathrm{m}$ in the frontal plane and stained with thionin) were available for the study of thalamic cytoarchitecture. 


\section{Results}

\section{Cytoarchitecture of the ventrolateral thalamus}

The terminology and subdivisions used to describe the ventrolateral thalamus are numerous and rather confusing (see Percheron, 1977, for a complete review). In order to facilitate the comparison of our results with those of other recent studies, we have employed the atlas and nomenclature of Olszewski (1952). In this section we will briefly describe the major features of the nuclei in the ventrolateral thalamus which were most heavily labeled following WGA-HRP injections into the SMA and APA. This analysis is hased on the study of sections from two normal animals and counterstained sections from the experimental animals. Although differences exist, our descriptions of this region are similar to those presented in several recent studies (e.g., Kusama et al., 1971; Percheron, 1977; Jones et al., 1979; Kalil, 1981; Asanuma et al., 1983a).

The relevant subdivisions of the ventrolateral thalamus are illustrated in Figures 1 to 4 . The most distinctive subdivision of the nucleus ventralis lateralis is the pars oralis (VLo). VLo is characterized by dense clusters of medium- to large-sized neurons. The large neurons within the clusters stain darkly for Nissl substance and have round or oval cell bodies. Between the clusters are regions of lower cell density, composed of fewer large neurons and smaller, less darkly stained neurons. Thus, the cytoarchitecture within VLo is not homogeneous.

Lateral to VLo is a strip of more loosely packed neurons which lacks dense cell clusters. This region has large, darkly staining neurons and is considered a rostral extension of the nucleus ventralis posterior lateralis, pars oralis (VPLo) (Fig. 2). The definition of this border is supported by our own connectional studies presented in the next section and those of others (e.g., Nauta and Mehler, 1966; Kusama et al., 1971; Kim et al., 1976; Percheron, 1977; Jones et al., 1979; Stanton, 1980; Kalil, 1981; DeVito and Anderson, 1982; Asanuma et al., 1983 a, b, c; see also "Discussion").

At caudal levels (Fig. 3), VPLo begins to expand medially and splits VLo into dorsal and ventral groups of cell clusters. Further caudally (Fig. 4), both the dorsal and ventral groups of VLo clusters are absent. The dorsal clusters are gradually replaced by smaller, less darkly staining neurons (Fig. 4, region labeled by question mark). Although this dorsal area lacks clusters of large neurons, it resembles the rostral cellular regions which are arranged between the VLo clusters and shares many of the connectional properties of VLo (see below).

The ventral border of VLo is formed by the pars medialis subdivision of the nucleus ventralis lateralis (VLm) (Figs. 1 to 3 ). There is a gradual change in cytoarchitectonic characteristics between these two subdivisions. However, the neurons in VLm are more spindle shaped than are VLo neurons and have their long axes oriented approximately parallel to the internal medullary lamina. Dorsally, neurons in VLm are densely packed and darkly staining like neighboring VLo neurons. These characteristics are less prominent in ventromedial parts of VLm.

The medial border of VLo is formed by the region termed "area X" by Olszewski (1952). Area X is charac- terized by small, irregularly shaped neurons, which are lightly stained and loosely packed (Figs. 1 to 4). In general, the cells in more medial regions of area X, along the internal medullary lamina, are smaller and more lightly stained than those in more lateral regions. At caudal levels where VLo clusters are no longer present, area X lies just medial to VPLo (Fig. 4). It can be distinguished from both VLo and VPLo because it lacks large, darkly stained neurons. At rostral levels, area X is replaced by Olszewski's two subdivisions of the nucleus ventralis anterior: pars magnocellularis (VAmc) and pars parvocellularis (VApc) (Fig. 1). The neurons in VAmc are larger and more darkly stained than those in area $\mathrm{X}$, whereas those in VApc are more clumped.

The dorsal border of VLo is formed caudally by the pars caudalis subdivision of the nucleus ventralis lateralis (VLc) (Figs. 2 to 4). VLc neurons are smaller and more lightly stained than neurons in both VLo and VPLo. In other respects, lateral VLc shares many of the cytoarchitectonic characteristics of adjacent regions of VPLo, whereas medial VLc has much in common with adjacent regions of area $\mathrm{X}$.

Later in the results we will describe the topographic distribution of thalamic neurons labeled by retrograde transport. These results support the cytoarchitectonic distinctions we make by demonstrating that distinct subdivisions of the ventrolateral thalamus contain labeled neurons following WGA-HRP injections into different cortical areas.

\section{Cortical projections from the ventrolateral thalamus}

We will present the plots from three animals with the most extensive cortical injections of WGA-HRP in order to give the most complete representation of thalamic inputs to the SMA, APA, and motor cortex. Figure $5 B$ illustrates the cortical injection sites ( small dots) and the spread of WGA-HRP (dashed lines enclosing the injection sites) for the three animals. The plots of labeled neurons found on single thalamic sections from the three animals are illustrated in Figures 7 to 9 . The sections in each of these figures are taken from comparable levels of the thalamus. The cytoarchitectonic borders were drawn based on thionin counterstaining of each section. Photomicrographs of thalamic labeling resulting from cortical injections of WGA-HRP are shown in Figures 6 and 10 to 13 .

In all animals, the cortical injections resulted in a

\footnotetext{
${ }^{1}$ The abbreviations used in the figures are: $A M$, nucleus anterior medialis, $A r S$, arcuate sulcus; $A V$, nucleus anterior ventralis; Cauds, nucleus caudatus; $C C$, corpus callosum; $C g s$, cingulate sulcus; $C S$, central sulcus; $C s l$, nucleus centralis superior lateralis; $D N c$, caudal deep cerebellar nuclei; $D N r$, rostral deep cerebellar nuclei; $I L$, inferior limb of the arcuate sulcus; $I P c S$, inferior precentral sulcus; $I p S$, intraparietal sulcus; $L D$, nucleus lateralis dorsalis; $M C$, motor cortex; $P c n$, $P C N$, nucleus paracentralis; $P f$, nucleus parafascicularis; $\operatorname{Pr} S$, principal sulcus; $R$, nucleus reticularis; $R e$, nucleus reuniens; $S f$, nucleus subfascicularis; Sf. $m c$, nucleus subfascicularis, pars magnocellularis; $S L$, superior limb of the arcuate sulcus; $S \Gamma c S$, superior precentral sulcus; Sub Thal, Sub T, subthalamic nucleus; TMT, tractus mamillo-thalamicus; $V P L c$, nucleus ventralis posterior lateralis, pars caudalis; $V P M$, nucleus ventralis posterior medialis; $V P M p c$, nucleus ventralis posterior medialis, pars parvocellularis; $X$, area $\mathrm{X}$; $Z i c, Z I$, zona incerta.
} 


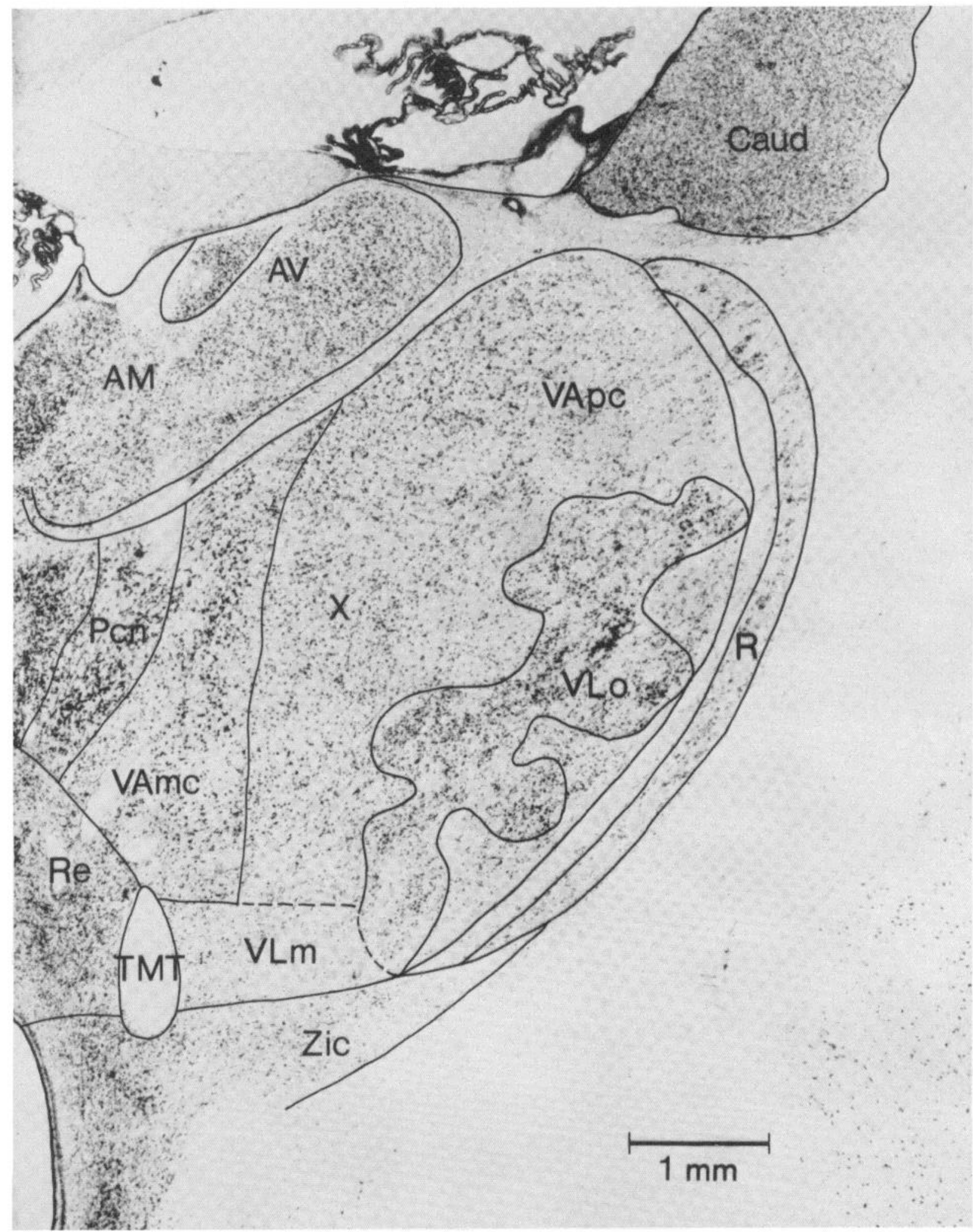

Figure 1. ${ }^{4}$ Photomicrograph of a thalamic section at the approximate level of A10.3. (Sections illustrated in Figs. 1 to 4 and $13 B=50 \mu \mathrm{m}$, thionin). The thalamic levels are according to Olszewski (1952). The unlabeled region lateral to VLo is a rostral extension of VPLo. See the text for further description and Footnotes 3 and 4 for abbreviations in this and the following figures.

"slab-like" region of thalamic labeling. The "slabs" of thalamic labeling extended for more than $6 \mathrm{~mm}$ rostrocaudally and up to $3 \mathrm{~mm}$ dorsoventrally. Each tended to be located more ventrally at rostral thalamic levels and more dorsally at caudal levels. The thalamic "slabs" which were labeled following the injections into the SMA and APA were relatively thin and measured more than 2 $\mathrm{mm}$ in width only at their greatest extent. 
Labeled neurons were not homogeneously distributed gions which either lacked or contained very few labeled throughout the thalamic slab which projected to a cortical area. Each slab of thalamic labeling was composed of small patches of labeled neurons intermingled with reneurons (Fig. 6A). The size of the labeled patches and the gaps between them varied at different rostrocaudal levels. The patches were smallest and the gaps between

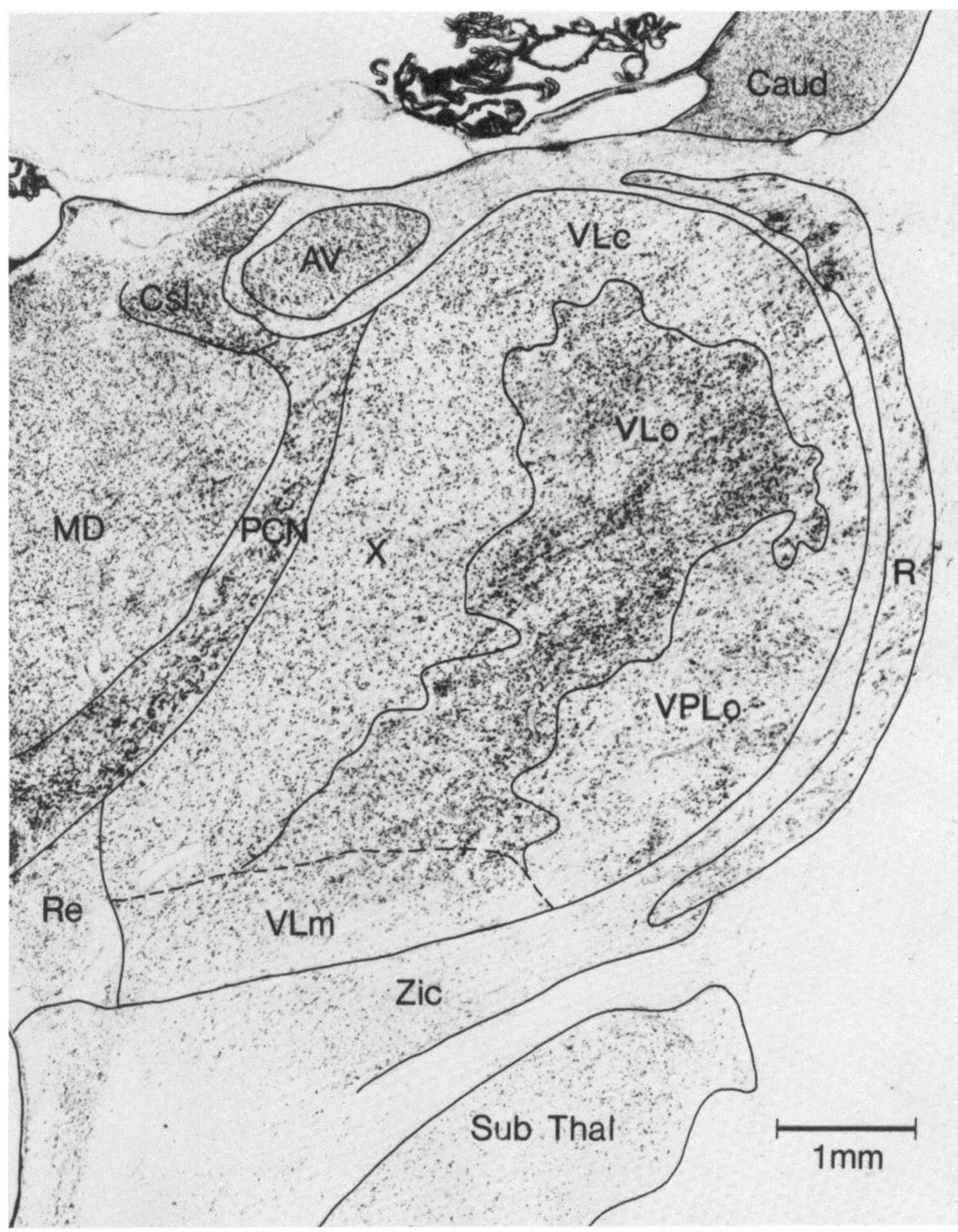

Figure 2. Photomicrograph of a thalamic section at the approximate level of A9.1. Note that VLo separates area X and VPLo. 


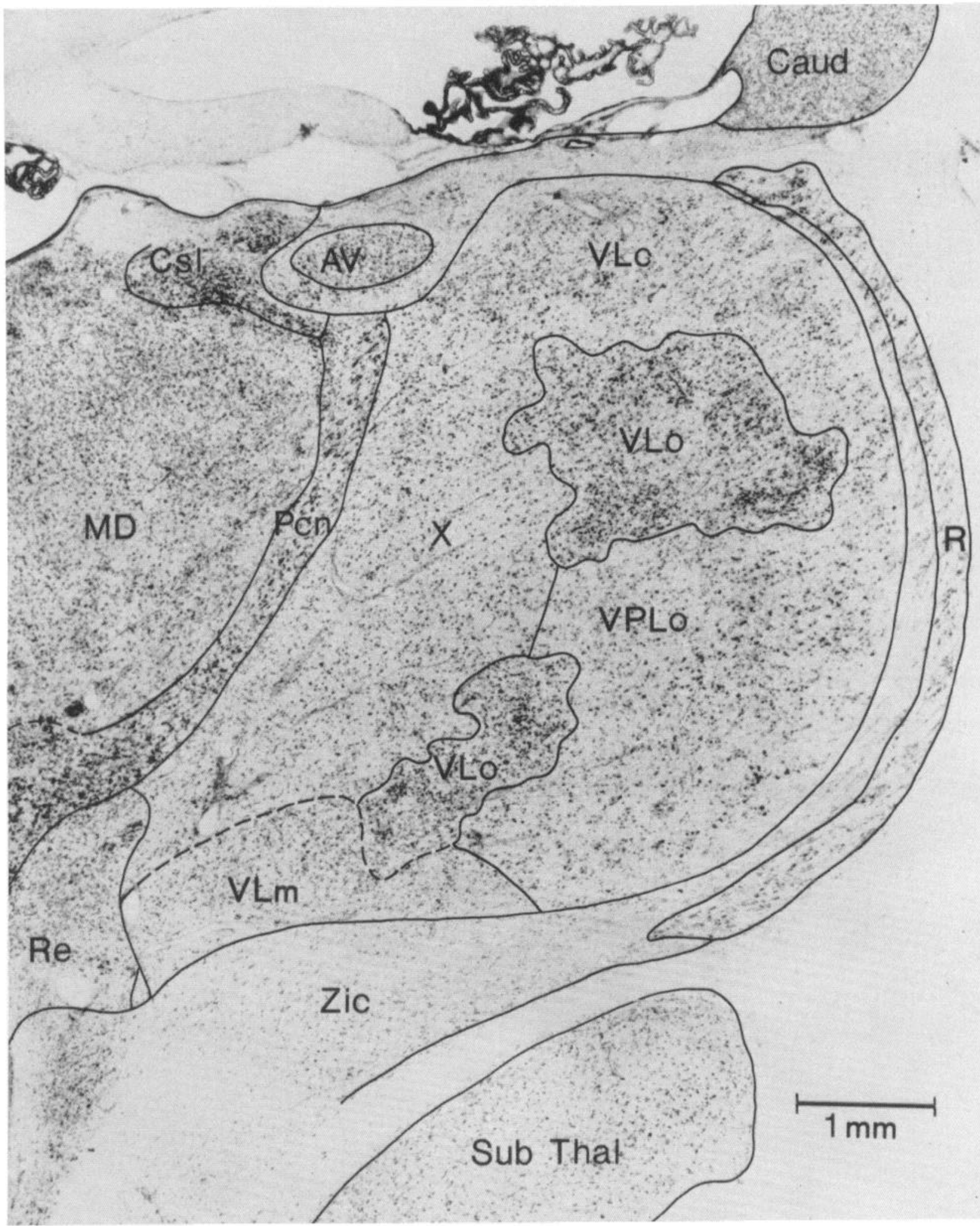

Figure 3. Photomicrograph of a thalamic section at the approximate level of A8.5. Note that VLo is split into dorsal and ventral clusters by the expansion laterally of VPLo.

them were largest at the rostral and caudal extremes of each labeled zone. Serial reconstruction of labeled patches in two animals demonstrated that they have an irregular "rod-like" shape. Individual "rods" can extend for up to $2.5 \mathrm{~mm}$ in the anterior-posterior axis. They coalesce to form larger accumulations of labeled neurons and then separate again into individual rods.

In addition to labeling neuron cell bodies by retrograde 
transport, the cortical injections of WGA-HRP also resulted in dense patches of punctate labeling in the ventrolateral thalamus (Fig. 6). Punctate labeling was also seen at many of the known sites of termination of various cortical efferents (e.g., thalamic reticular nucleus (Fig.
$6 B)$, caudate, putamen, pontine nuclei, and regions surrounding the red nucleus). Since these regions do not project directly to the cortex and lacked cell bodies labeled by retrograde transport, it is likely that much of the punctate labeling outside of neuron cell bodies rep-

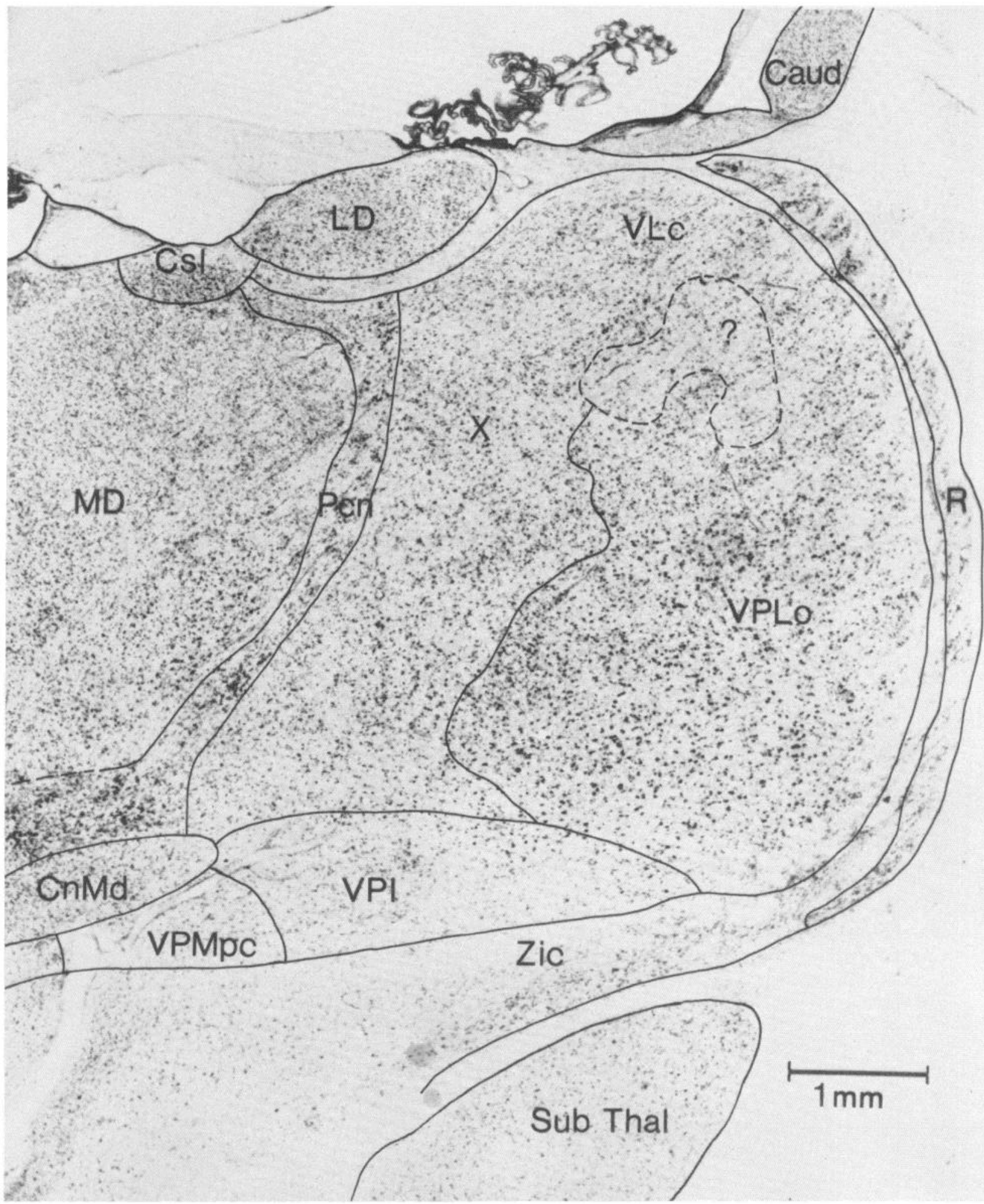

Figure 4. Photomicrograph of a thalamic section at the approximate level of A7.7. VLo clusters are absent at this level. The dorsal cluster is replaced by a parvocellular region which is indicated by the question mark. 
A

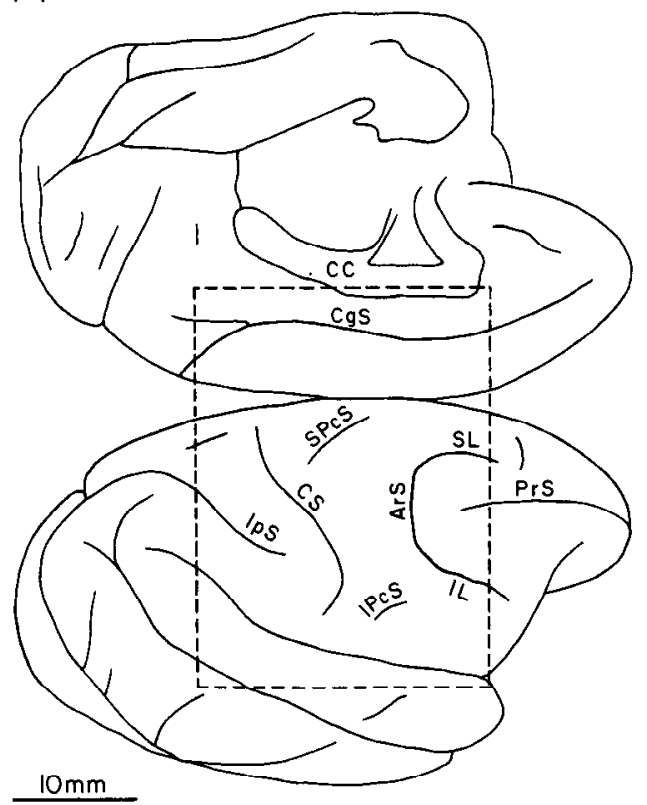

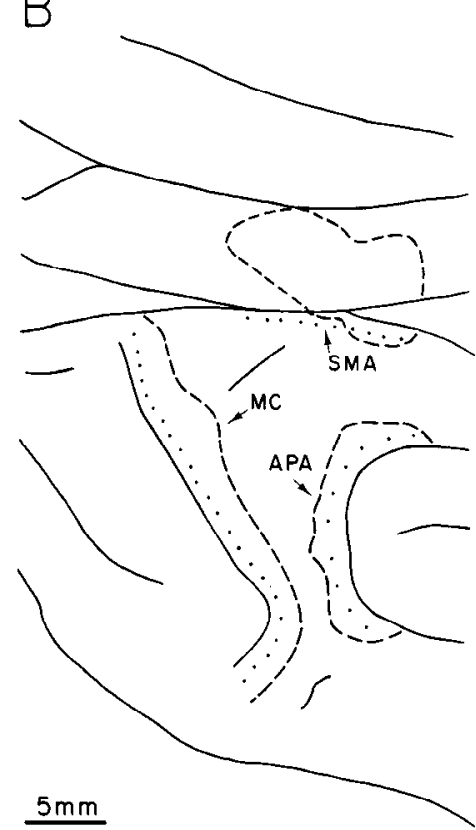

Figure 5. Location of cortical injection sites. $A$, Two views of the cortical surface from the right hemisphere of a rhesus monkey. Lateral view (bottom) and medial view reflected upward (top). $B$, Enlarged view of the cortical area included within the dashed rectangle of $A$. The cortical injection sites (small dots) and the spread of WGA-HRP reaction product (dashed lines) for the three animals are plotted on a single hemisphere. The motor cortex $(M C)$ is adjacent to the rostral bank of the central sulcus $(C S)$. The SMA is near the medial wall of the hemisphere. The APA is adjacent to the caudal bank of the arcuate sulcus $(A r S)$.

resents anterograde axonal transport of WGA-HRP (see Mesulam, 1982, for a more complete discussion). In the ventrolateral thalamus, there was a close correspondence between the size, shape, and distribution of the patches of labeled cell bodies and the patches of punctate labeling (Fig. 6A). Furthermore, punctate labeling was sparse in the cellular regions between the patches of labeled cell bodies. This suggests that the patches of cell bodies in the ventrolateral thalamus which are the origin of thalamic projections to a cortical area are also the recipients of a focused corticothalamic input.

Injections of WGA-HRP into the two premotor areas and the motor cortex also led to anterograde and retrograde labeling in various cortical areas. For example, APA injections resulted in labeling in the SMA and motor cortex; SMA injections resulted in labeling in the APA and motor cortex; and motor cortex injections resulted in labeling in the APA and SMA. Thus, the APA, SMA, and motor cortex are reciprocally interconnected. A full description of these and other intracortical connections which were observed will be presented in a subsequent publication.

\section{Origin of thalamic input to the supplementary motor area (SMA)}

In order to label the origin of thalamic input to the SMA, injection sites were placed along the medial wall of the hemisphere at the points shown in Figure $5 B$. The spread of WGA-HRP included the dorsal bank of the cingulate sulcus only caudally. At this point, reaction product was more than $8 \mathrm{~mm}$ from the level of the central sulcus. According to various maps of motor representation in the SMA (e.g., Woolsey et al., 1952; Brinkman and Porter, 1979b; Matsumura and Kubota, 1979; Muakkassa and Strick, 1979; Wise and Tanji, 1981; Tanji and Kurata, 1982), the spread of WGA-HRP included most of the "forelimb representation" and extended to parts of the "face representation" rostrally and the "hindlimb representation" caudally.

Plots of representative thalamic sections from an animal with injections into the SMA (sites illustrated in Fig. $5 B$ ) are shown in Figure 7. Photomicrographs of thalamic sections from another animal with injections into the SMA are presented in Figure 10. These figures illustrate that injections into the SMA resulted in a slab of labeled neurons which was principally located in VLo. Thalamic labeling was most extensive between Olszewski's levels A10.5 and A8.5 where VLo is most extensive. In many instances, changes in the location of labeled neurons paralleled cytoarchitectonic changes in VLo. For example, as VLo split into dorsal and ventral cell groups, labeled neurons also split into dorsal and ventral groups (Fig. 7, section 347). Patches of labeled neurons were found in both the cluster and non-cluster regions of VLo.

The slab of labeled neurons extended into the most dorsal aspects of adjacent VLm and the dorsal, parvocellular region just caudal to VLo (Figs. 7, section 372, and $10 D$ ). Thalamic labeling extended as far caudally as A3.0 


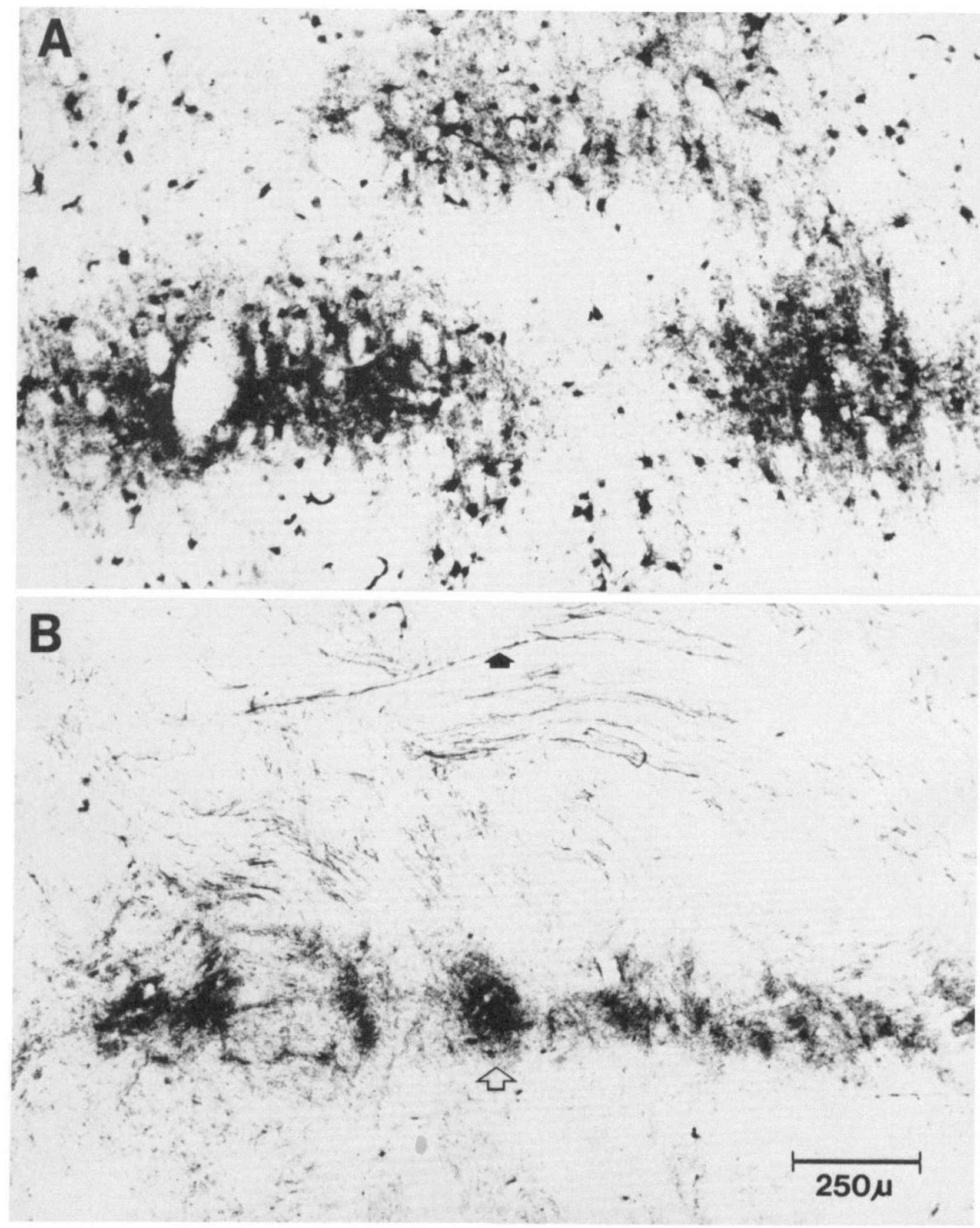

Figure 6. Retrograde and anterograde labeling in the thalamus. A, Retrograde and anterograde labeling in area $\mathrm{X}$ following a WGA-HRP injection into the APA. Note the discrete patches containing both types of labeling. The magnification in $A$ is the same as that in $B$. $B$, Anterograde labeling in and adjacent to the reticular nucleus. The open arrow points to a patch of punctate labeling indicative of anterograde transport. Note the absence of labeled cell bodies. The solid arrow points to a labeled axon in the internal capsule. 

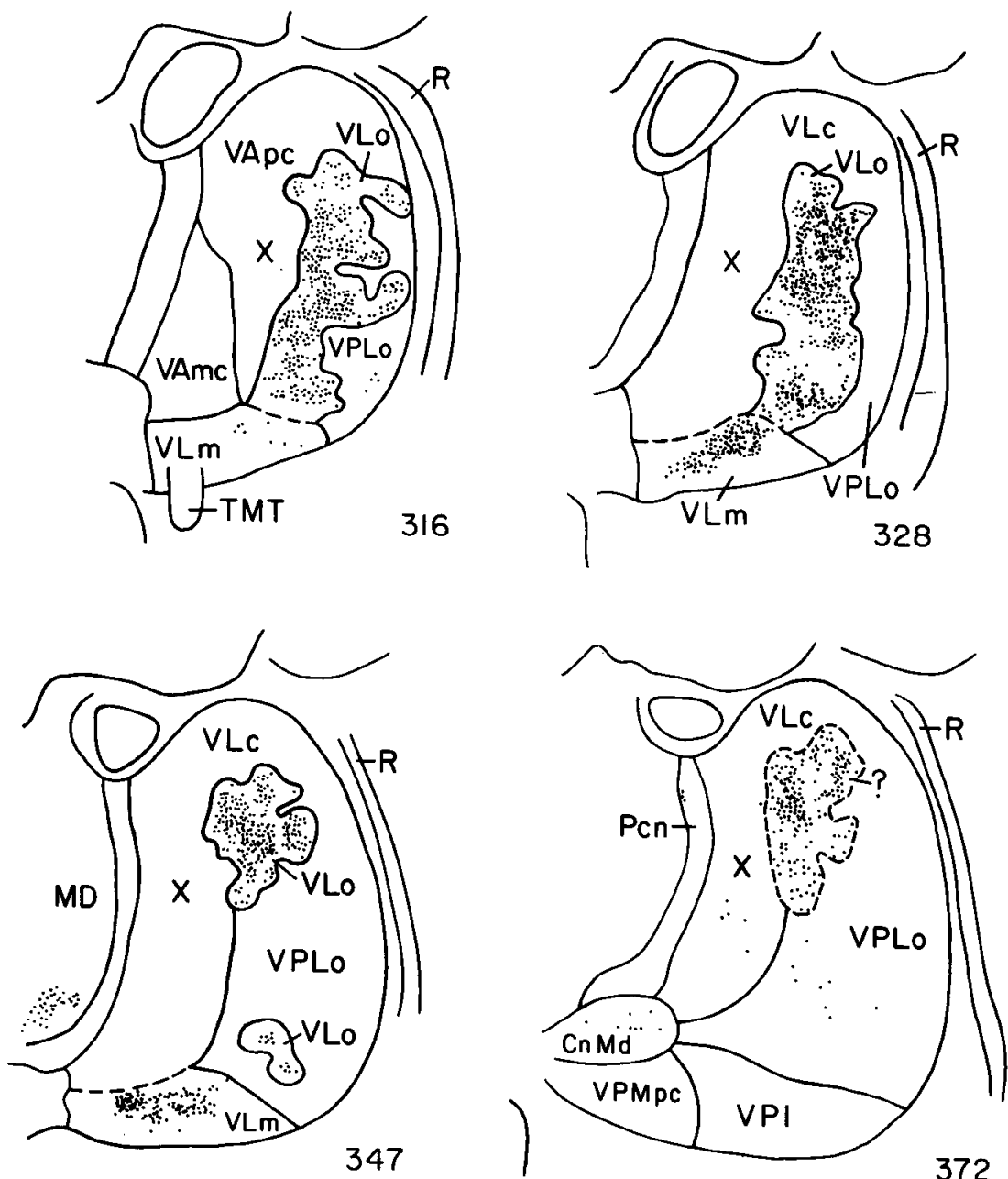

347

372

Figure 7. Plots of labeled neurons resulting from WGA-HRP injections into the SMA. Labeled neurons found on single sections at different levels of the thalamus are indicated by small dots in Figures 7 to 9 . The injection sites in the SMA near the medial wall of the hemisphere are shown in Figure $5 B$. The sections chosen for illustration in Figures 7 to 9 are at approximately the same level as those used to illustrate thalamic cytoarchitecture in Figures 1 to 4 .

where a cluster of labeled neurons was found in the ventral portion of the nuclear subdivision termed pars postrema (VLps) by Olszewski (1952). This labeling was located just dorsal to nucleus lateralis posterior.

\section{Origin of thalamic input to the arcuate premotor area (APA)}

In order to label the origin of input to the APA, injection sites were placed $1 \mathrm{~mm}$ posterior to the caudal bank of the arcuate sulcus at the points shown in Figure $5 B$. WGA-HRP spread no more than $2 \mathrm{~mm}$ from any of the injection sites. Thus, some reaction product was found in the caudal bank of the arcuate sulcus, but none was seen in the rostral bank. According to various maps of motor representation in the APA (e.g., Kubota and Hamada, 1978; Matsumura and Kubota, 1979; Muakkassa and Strick, 1979; Weinrich and Wise, 1982), the spread of WGA-HRP included most of the "forelimb representation" located around the spur of the arcuate sulcus, some of the "face representation" near the inferior limb of the sulcus, and some of the "hindlimb representation" near the superior limb of the sulcus.
Plots of representative thalamic sections from the animal with the injections into the APA (sites illustrated in Fig. $5 B$ ) are shown in Figure 8. Photomicrographs of thalamic sections from this animal are presented in Figures 11 and $13 B$. Injections of WGA-HRP into the APA resulted in a slab of labeled neurons which was located principally in area $\mathrm{X}$. The slab of labeled neurons was most extensive between Olszewski's levels A9.5 and A7.5. The labeling in area $\mathrm{X}$ was just medial to the thalamic labeling which was seen after injections into the SMA. Only the medial aspect of area X, adjacent to the internal medullary lamina, lacked substantial numbers of labeled neurons. Thalamic labeling following the APA injections extended dorsally into contiguous areas of medial VLc and rostrally into the dorsomedial aspect of rostral VLm. Labeled neurons were found as far caudally as A3.0 where a small cluster of labeled neurons was seen in VLps. These labeled neurons were located just dorsal to the labeling seen after SMA injections.

In one animal, injection sites were placed around the spur of the arcuate sulcus in the "arm area" of the APA (e.g., Kubota and Hamada, 1978; Matsumura and Ku- 
bota, 1979; Muakkasa and Strick, 1979; Weinrich and Wise, 1982). The spread of reaction product in this animal was more confined than that observed in the animal illustrated in Figure $5 B$. The more localized injections resulted in thalamic labeling which was less extensive than that illustrated in Figures 8, 11, and $13 B$. As in the animal illustrated, labeled neurons were largely confined to area X. However, the slab of labeling was thinner and contained fewer patches of labeled neurons. In addition, labeled patches were laterally located in area $\mathrm{X}$ and did not extend as far dorsally or ventrally. Less of VLc and VLm was labeled in this animal than was labeled in the animal with the more extensive injections.

\section{Origin of input to the motor cortex from rostral regions of the ventrolateral thalamus}

We next sought to determine whether there is any overlap between the regions of the ventrolateral thalamus which project to the SMA and APA and the region which projects to the motor cortex. Because the preceding analysis had shown that the two premotor areas received their input from rostral parts of the ventrolateral thalamus, we concentrated our analysis on this area. In order to label the origin of thalamic input to the motor cortex, injection sites were placed $1 \mathrm{~mm}$ rostral to the central sulcus at the points shown in Figure $5 B$. WGAHRP spread 2 to $3 \mathrm{~mm}$ from the injection sites. The rostral bank of the central sulcus (upper one-third) contained reaction product throughout its mediolateral extent. Reaction product spread into somatic sensory cortex in two regions: medially, where the "hindlimb representation" is located, and laterally, where the "face representation" is located. According to various maps of body representation in the motor cortex (e.g., Woolsey et al., 1952; Kwan et al., 1978), the spread of WGA-HRP involved largely the representation of distal limb parts and those parts of the "face" and "proximal body representations" which are adjacent to the central sulcus.

Plots of representative thalamic sections from the animal with injections into the motor cortex are shown in Figure 9. Photomicrographs of thalamic sections from this animal are presented in Figure 12. Injections of WGA-HRP into the motor cortex resulted in labeling which was largely confined to VPLo, particularly at rostral thalamic levels. The slab of labeled neurons filled VPLo and extended up to its borders with area X and VLo. No labeled neurons were found in area X or VLo following injections into the motor cortex. Scattered labeling extended across the ventral border of VPLo (Figs. 9 and 12) into the nucleus labeled ventralis pos-
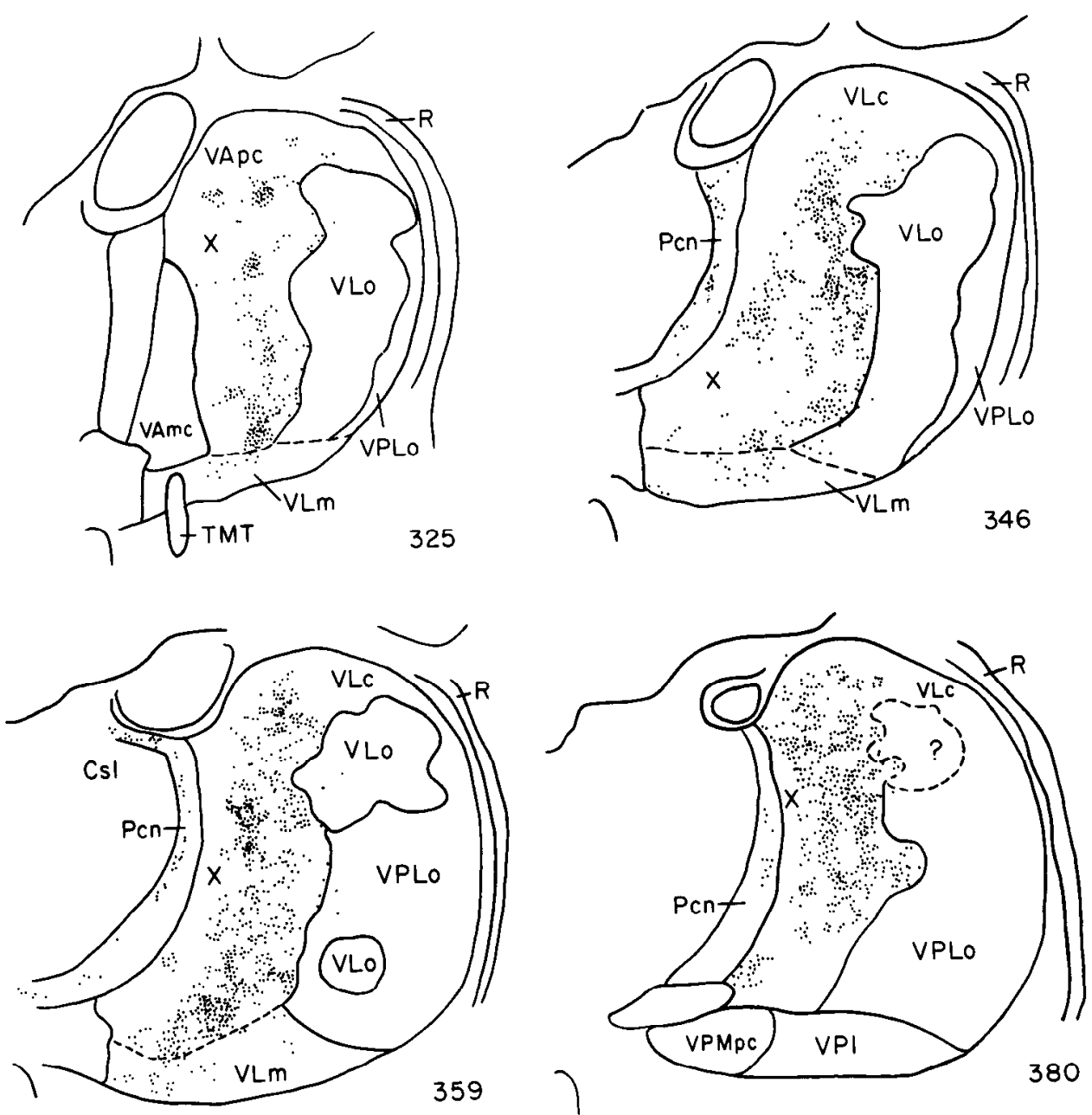

Figure 8. Plots of labeled neurons resulting from injections into the APA. The injection sites in the APA near the caudal bank of the arcuate sulcus are shown in Figure $5 B$. 

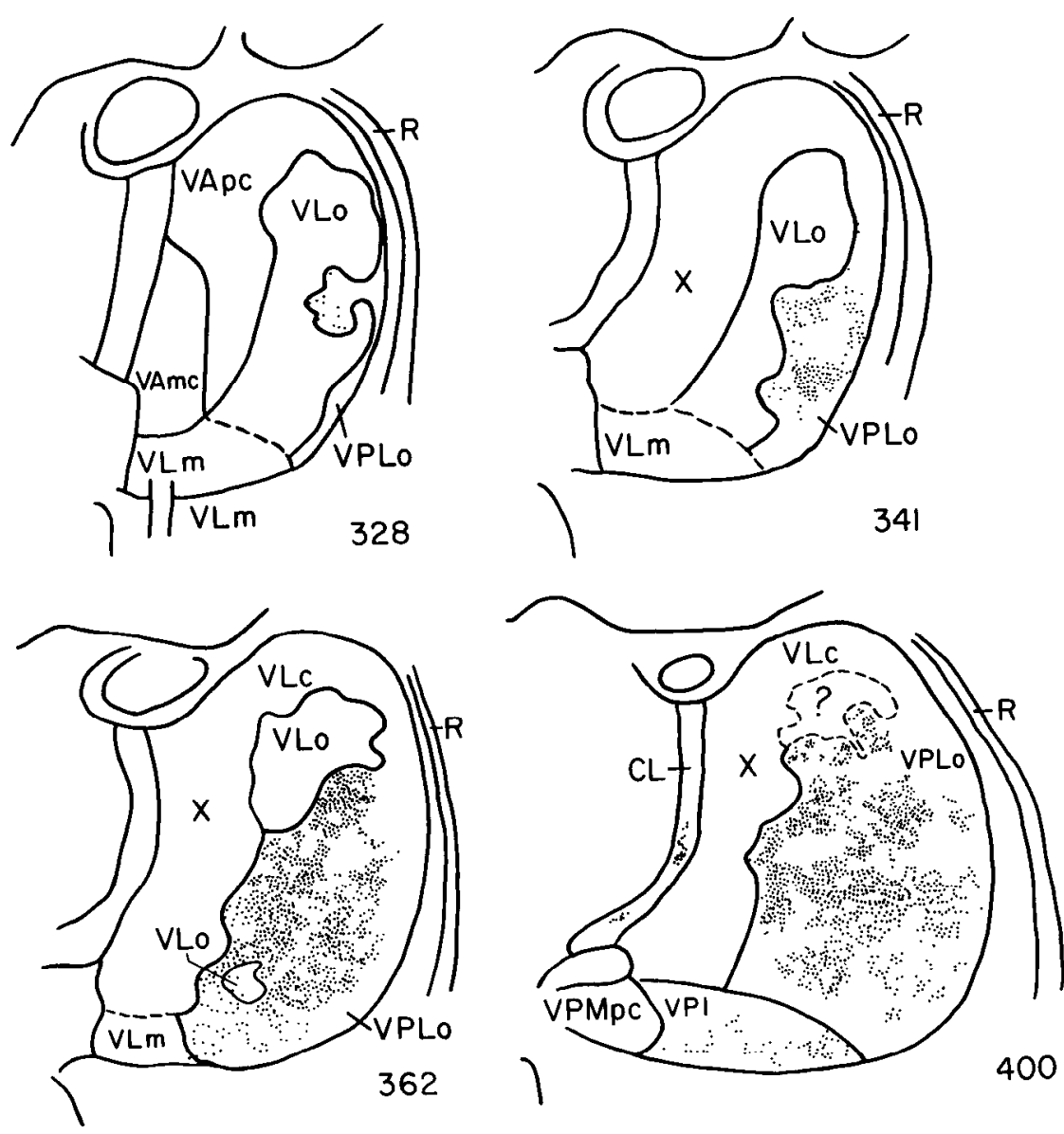

Figure 9. Plots of labeled neurons resulting from injections into the motor cortex. The injection sites in the motor cortex near the rostral bank of the central sulcus are shown in Figure $5 B$. terior inferior (VPI) by Olszewski (1952). Whether the labeling in VPI was the result of transport from the motor cortex or was due to spread of reaction product to adjacent somatic sensory cortex (see above) could not be determined from our studies.

\section{Additional thalamocortical projections}

Most of the thalamic labeling following cortical injections into the SMA and APA was located in various subdivisions of the ventrolateral thalamus. However, additional labeling, indicative of anterograde and retrograde transport, was seen in isolated parts of the nucleus medialis dorsalis (MD) (Figs. 7, section 317; 10D; and $13 B)$ and three intralminar nuclei: centralis lateralis (CL) (Figs. 9, section $400 ; 10 D$; and $13 B$ ), paracentralis (Pcn) (Fig. 8), and centrum medianum (Cn Md) (Figs. $11 B$ and $13 B$ ). Some of the labeling in MD and adjacent CL was both particularly dense and topographically organized. Since these thalamic regions were not the focus of this study, we will describe only the most obvious patterns of labeling.

The typical patterns of labeling seen in MD and CL following SMA and APA injections are shown in Figures $10 D$ and $13 B$. Note that labeling in the caudal portions of the ventrolateral thalamus is also present on these sections. Figure $13 A$ is a Nissl-stained section at a thalamic level comparable to that shown in Figures $10 \mathrm{D}$ and $13 B$ (i.e., A5.7, according to Olszewski, 1952). Following SMA and APA injections, labeling in MD and CI, was observed at approximately the same thalamic level. The
APA injections resulted in labeling which was slightly more dorsal in MD than that observed following SMA injections. The labeling in CL following the APA injections was more rostral and medial than that observed following the SMA injections. Injections into motor cortex resulted in labeling of more caudally located regions in $\mathrm{CL}$ and the most ventrolateral portion of caudal $\mathrm{MD}$ which overlies Cn MD (not illustrated). Thus, our evidence suggests that projections from $\mathrm{MD}$ and CL to motor and premotor areas originate from separate regions of these nuclei.

\section{Discussion}

We have employed retrograde transport of WGA-HRP to determine the origin of thalamic input to two of the premotor areas in the frontal lobe: the SMA and the APA. For the purpose of comparison, the origin of thalamic input to motor cortex regions adjacent to the central sulcus also was examined. We were particularly concerned with confining the spread of WGA-HRP to a single functional area. Our injections into the SMA and APA were focused on those regions which project to the motor cortex (e.g., Pandya and Kuypers, 1969; Jones and Powell, 1970; Pandya and Vignolo, 1971; Matsumura and Kubota, 1979; Muakkassa and Strick, 1979) and which possess neurons active during movement (e.g., Kubota and Hamada, 1978; Brinkman and Porter, 1979a, b; Smith 1979; Tanji et al., 1980; Wise and Tanji, 1981; Weinrich and Wise, 1982). Thus, while we have not attempted to examine the full extent of the thalamic 


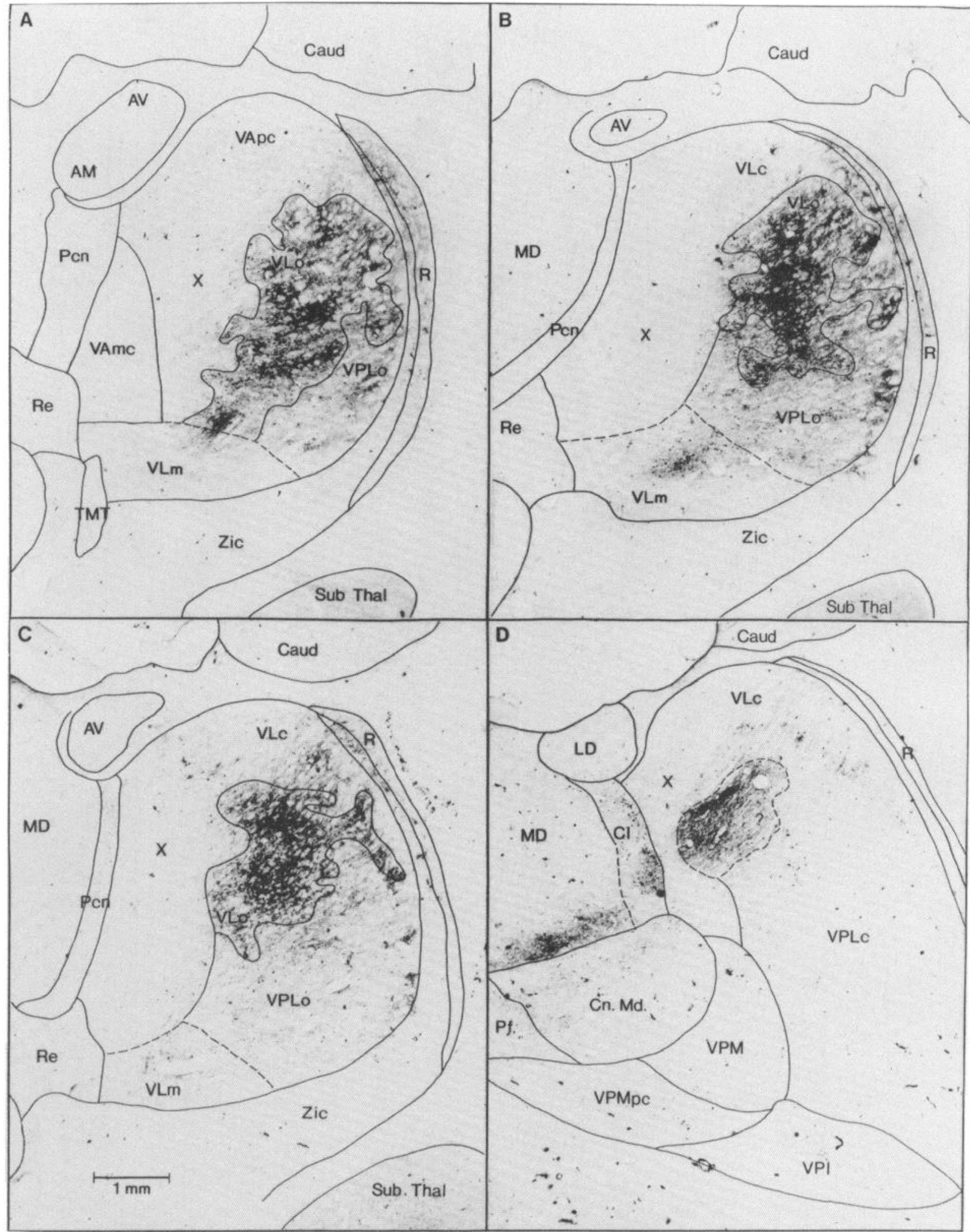

Figure 10. Photomicrographs of thalamic labeling resulting from injections into the SMA. The injection sites for this animal were comparable to the SMA sites illustrated in Figure $5 B$. The sections illustrated in Figures 10 to $13 B$ were photographed uncounterstained. The coverslips were then removed and each section was counterstained with thionin. The borders of the thalamic subdivisions were then added to the photographs. The scale for each section is shown in $C$. 


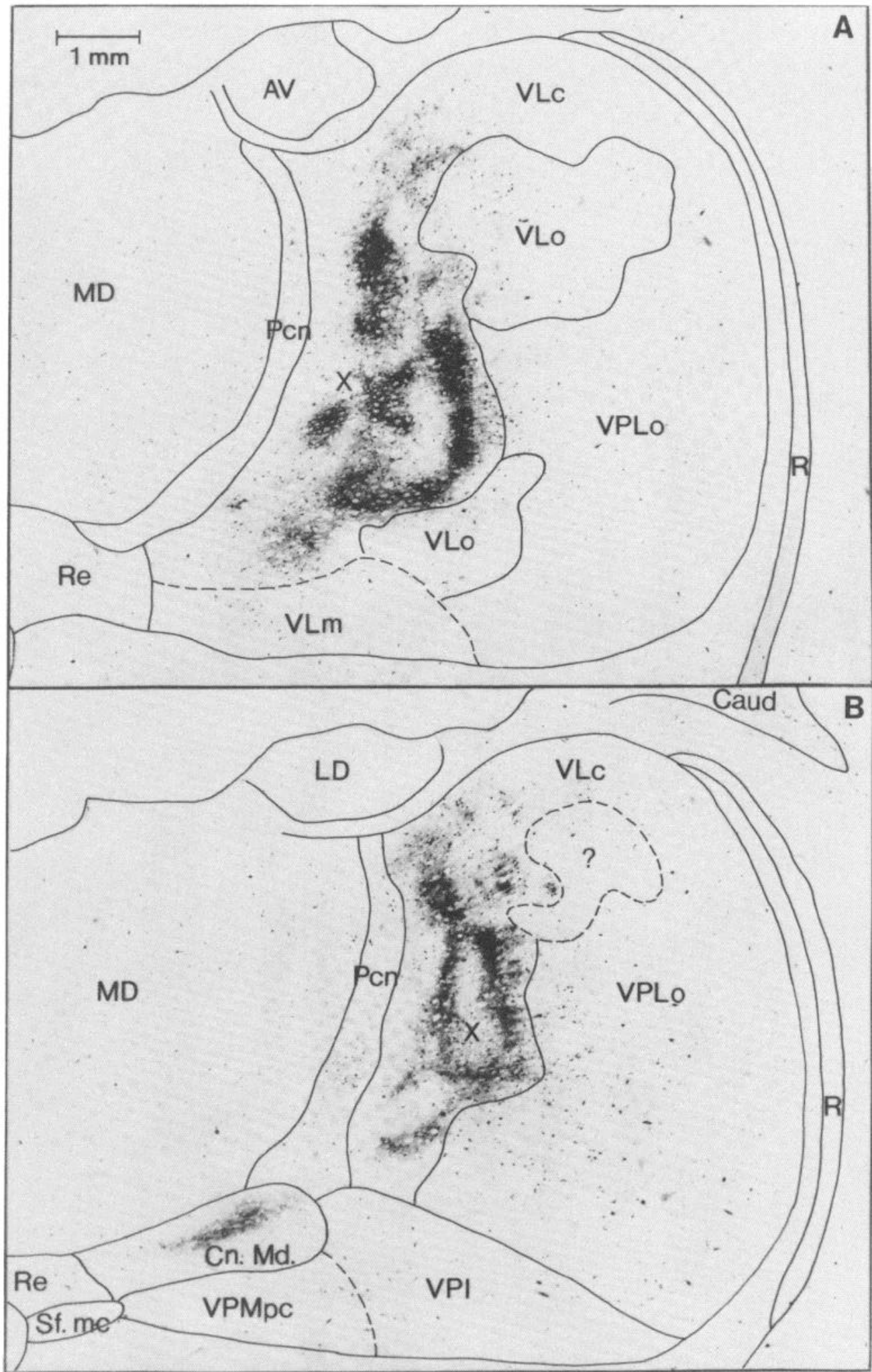

Figure 11. Photomicrographs of thalamic labeling resulting from injections into the APA. The injection sites for this animal are illustrated in Figure $5 B$. The scale for each section is shown in $A$.

input to each premotor area, our goal was to identify a major component of this input.

Injections of WGA-HRP into each cortical area resulted in dense, slab-like accumulations of labeled neu- rons in the ventrolateral thalamus. Injections into the APA resulted in thalamic labeling which was most dense in area X. In contrast, injections into the SMA resulted in thalamic labeling which was most dense in VLo. As in 


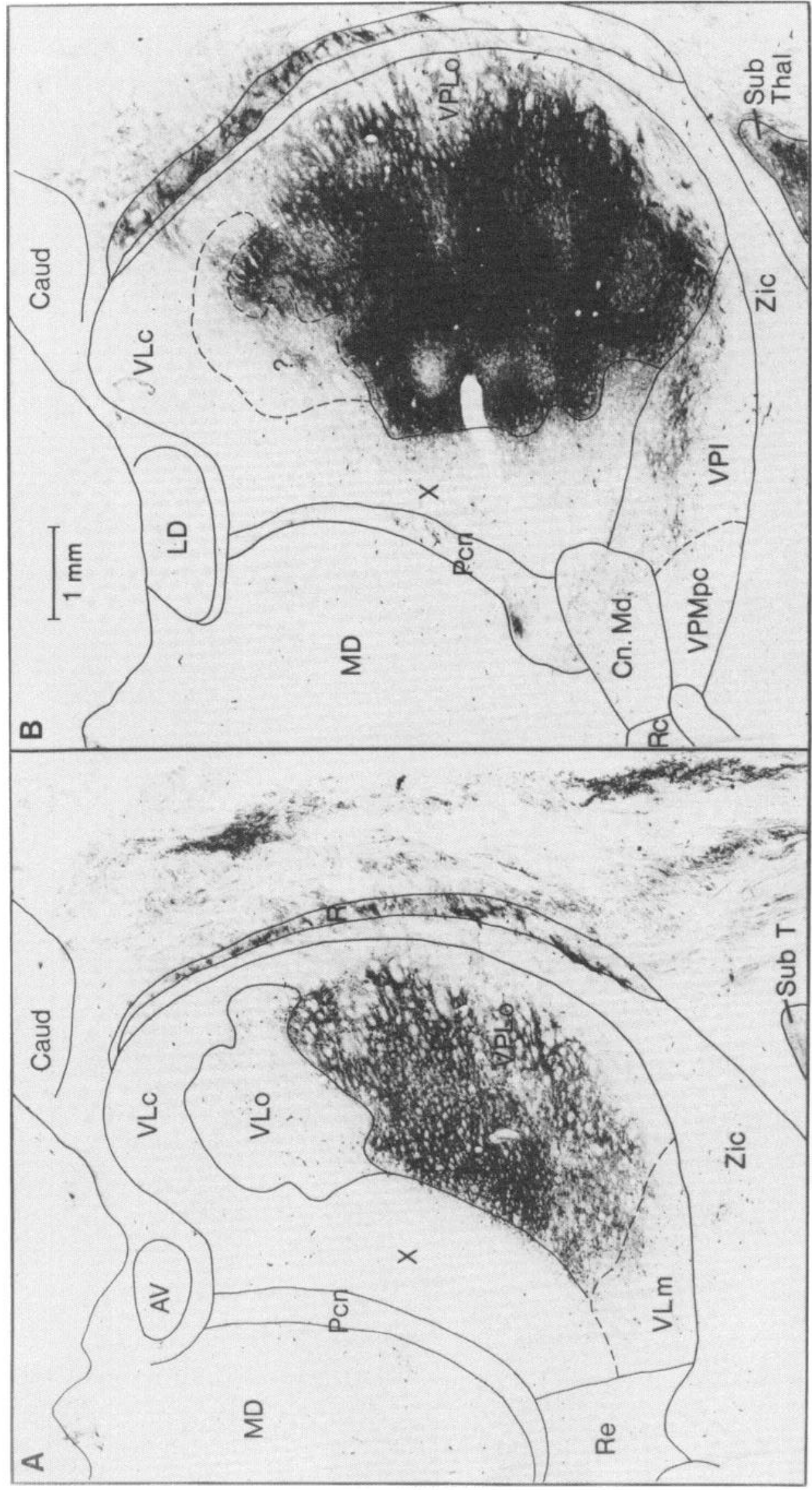

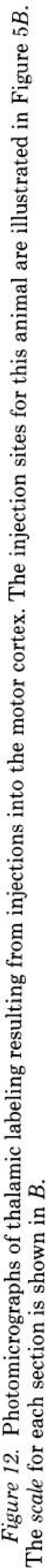



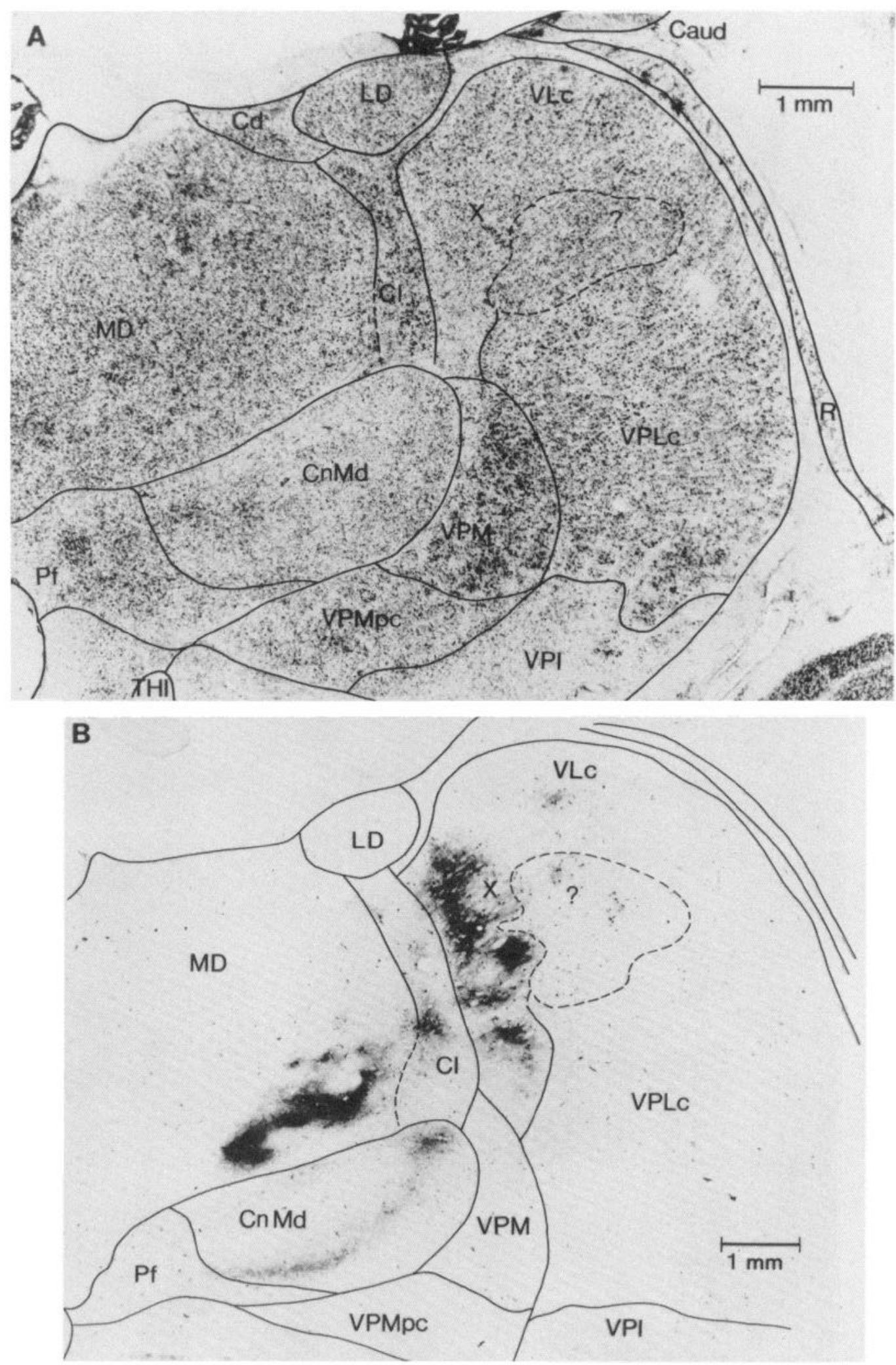

Figure 13. Photomicrographs of thalamic labeling and normal cytoarchitecture at comparable thalamic levels. A, Photomicrograph of a thalamic section at the approximate level of A5.7. Note the presence of area X and the parvocellular zone (indicated by the question mark) at this caudal thalamic level. $B$, Photomicrograph of thalamic labeling resulting from injections into the APA. The injection sites for this animal are illustrated in Figure $5 B$. Note that $\mathrm{Cl}, \mathrm{MD}$, and $\mathrm{Cn} \mathrm{Md}$ also are labeled in addition to area X. 
previous studies (Strick, 1975, 1976; Kievit and Kuypers, 1977; Jones et al., 1979), VPLo was most densely labeled following injections into the motor cortex. Thus, the two premotor areas and the motor cortex each received thalamic input from separate, cytoarchitectonically distinct subdivisions of the ventrolateral thalamus. In general, these conclusions are supported by the results of prior studies (e.g., SMA: DeVito and Smith, 1959; Wiesendanger et al., 1973; Kievit and Kuypers, 1977; Kalil, 1978; Kunzle, 1978; Brinkman and Porter, 1979b; Bowker et al., 1979; APA: Roberts and Akert, 1963; Akert, 1964; Kievit and Kuypers, 1977; Kunzle, 1978).

Injections into the SMA and APA also led to topographically organized labeling in MD. SMA injections labeled ventral and lateral regions of $\mathrm{MD}$, whereas regions of MD just dorsal to this were labeled following APA injections. At this time, it is not possible to determine the functional significance of the labeling in MD. It may represent either a spatially displaced continuation of the labeling in the ventrolateral thalamus (Kievit and Kuypers, 1977) or a functionally separate svstem of projections from the thalamus to each of the two premotor areas. Additional projections to the premotor areas also originated from well defined regions of the intralaminar nuclei.

\section{Afferent input to the ventrolateral thalamus}

As noted in the introduction, considerable information has recently become available regarding the differential patterns of cerebellar and pallidonigral termination in subdivisions of the ventrolateral thalamus. The significance of these patterns of thalamic termination has not been completely understood because the precise cortical projection of each thalamic subdivision was previously unknown. The present study identifies some of the cortical projections of these thalamic subdivisions. In the remainder of this discussion we will review some of the recent primate studies on the sites of pallidonigral and cerebellar termination in subdivisions of the ventrolateral thalamus and suggest how various thalamic subdivisions link subcortical motor nuclei with motor and premotor cortical areas.

\section{Basal ganglia projections to the ventrolateral thalamus}

The globus pallidus and the substantia nigra are the major output nuclei of the basal ganglia (for a complete review, see DeLong and Georgopoulos, 1981). The results of some recent studies on the sites of termination of efferents from the internal segment of the globus pallidus (GPi) upon the ventrolateral thalamus are illustrated in Figure $14, A$ to $D$. Although there are some minor differences between studies, all emphasized that efferents from GPi project upon three subdivisions of the ventrolateral thalamus: VLo, parts of the VApc, and VLm (Nauta and Mehler, 1966; Kuo and Carpenter, 1973; Kim et al., 1976; DeVito and Anderson, 1982).

The sites of termination of the substantia nigra in subdivisions of the ventrolateral thalamus have been examined extensively by Carpenter and his colleagues (Carpenter and McMasters, 1964; Carpenter and Strominger, 1967; Carpenter and Peter, 1972; Carpenter et al., 1976, 1981). The results of these studies are summarized

\section{Pallidal-Nigral Input}
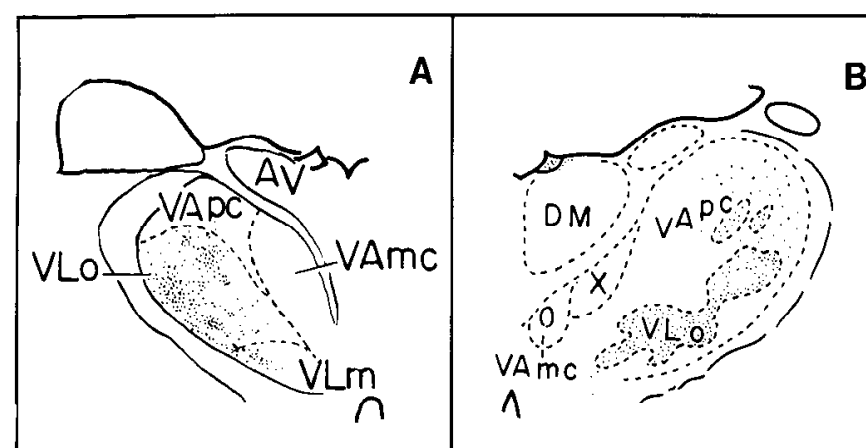

Kuo \& Carpenter, 1973

Kim, et. al., 1976

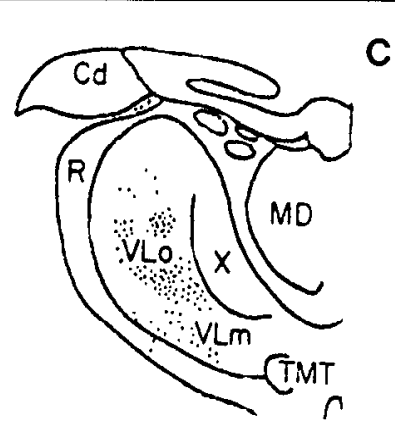

DeVito \& Anderson, 1982

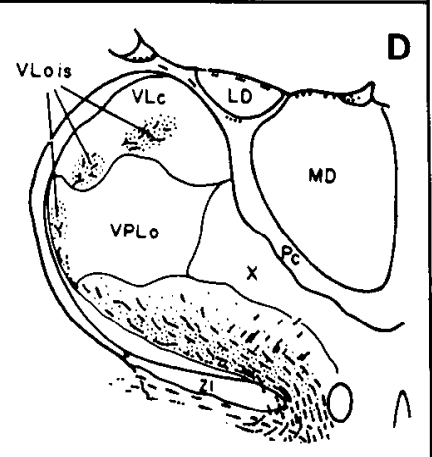

DeVito \& Anderson, 1982

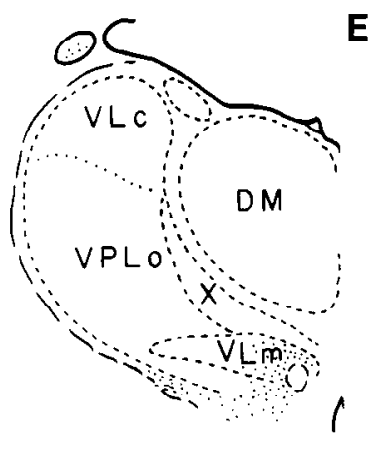

E

Nauta \& Mehler, 1966

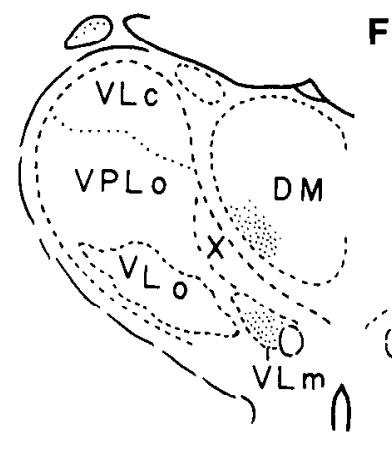

Carpenter, et. al., 1976

Figure 14. Pattern of pallidonigral termination in ventrolateral thalamus. $A$ to $D$ represent the sites of termination for pallidal efferents in the ventrolateral thalamus taken from the studies of Kuo and Carpenter (1973), Kim et al. (1976), DeVito and Anderson (1982), and Nauta and Mehler (1966). $E$ and $F$ represent the sites of termination for nigral efferents in the ventrolateral thalamus taken from the work of Carpenter et al. (1976). The levels of the sections chosen for illustration correspond to the levels of the normal and experimental material illustrated in Figures 1 to 4, 7, and 10 .

in Figure $14, E$ and $F$. Nigrothalamic projections originate from the pars reticulata segment of the substantia nigra (SNpr) and terminate in two subdivisions of the ventrolateral thalamus: VAmc and VLm. Additional projections also terminate in paralaminar MD. A comparison of Figure 14, $A$ to $D$ with Figure 14, $E$ and $F$ indicates the lack of overlap between nigral and pallidal sites of termination in the thalamus. 
These patterns of pallidothalamic and nigrothalamic projections have led to the generally accepted conclusion that the pallidonigral system most directly influences areas 4 and 6, and even prefrontal cortical areas (Brodal, 1981; DeLong and Georgopoulos, 1981). However, our results suggests a different scheme of organization. First, a comparison of the results of the present study (Figs. 7 and $10, A$ to $C$ ) with the sites of pallidothalamic termination (Fig. 14, $A$ to $D$ ) indicates that part of the pallidal output is focused on subdivisions of the ventrolateral thalamus which project to the SMA (i.e., VLo and VLm). Second, a comparison of our results (Figs. 7, sections 328 and 347 , and $10 B$ ) with Carpenter's studies (Fig. 14, E and $F$ ) indicates that part of the nigral output also is focused on thalamic regions which project to the SMA (i.e., VLm and MD). Third, neither pallidal nor nigral efferents appear to terminate in thalamic regions which project to the APA or the motor cortex.

Recent studies suggest that SNpr and GPi should be considered a functional unit (DeLong and Georgopoulos, 1979). This concept is based on physiological observations in awake primates which demonstrate: (1) similar discharge rates and firing patterns of neurons in GPi and SNpr, and (2) a somatotopic organization of GPi which continues into SNpr. Neurons related to orofacial movements were located on both sides of the internal capsule in the lateral portion of $\mathrm{SNpr}$ and the ventrocaudal segment of GPi. The two nuclei also show similarities in their anatomical organization (for discussion and references see Brodal, 1981; DeLong and Georgopoulos, 1981; DeLong et al., 1983). The present study further supports this concept by demonstrating that the thalamic regions which receive efferents from $\mathrm{SNpr}$ and $\mathrm{GPi}$ project to a common cortical target.

As noted above, the sites of termination of pallidal and nigral efferents do not overlap in the thalamus. We would like to suggest that the lack of overlap may indicate that the somatotopic organization of the pallidonigral system is maintained in its thalamic projections. If this is the case, then the "face" representation in SNpr may project to a "face" representation in VLm which in turn may project to the "face" representation in the SMA. Likewise, "arm and leg" representations in GPi may project to "arm and leg" representations in VLo which in turn may project to "arm and leg" representations in the SM $\Lambda$. These suggestions must be explored by further experiments which examine the somatotopic organization of both pallidonigral inputs to the thalamus and thalamocortical inputs to the SMA.

Not all of the outputs from the pallidonigral system are focused on the SMA. The cortical projections of VApc, which receives pallidal input, and VAmc, which receives nigral input, have not been well defined but appear to project to cortical regions rostral to the premotor areas (Carmel, 1970; Kievit and Kuypers, 1977; Kunzle, 1978). Furthermore, the existence of eye movement-related neurons in SNpr suggests that there may be additional nigral efferents to thalamic regions which are concerned with oculomotor control (IIikosaka and Wurtz, 1983). However, our results indicate that, of the cortical areas concerned with limb movement, the pallidonigral system is most directly connected to the SMA.

\section{Cerebellar projections to the ventrolateral thalamus}

Cerebellothalamic projections originate from all three deep cerebellar nuclei: dentate, interpositus, and fastigial (see Brooks and Thach, 1981; Asanuma et al., 1983b, c, for recent reviews). The results of some recent primate studies on the sites of termination of cerebellothalamic efferents are illustrated in Figure 15. Although studies differ in the nomenclature and borders applied to each nucleus, and in the techniques employed, they show remarkable agreement concerning the regions of ventrolateral thalamus which receive cerebellar input. The deep nuclei terminate in four subdivisions of the ventrolateral thalamus: VPLo, area X, VLc, and VLps (Kusama et al., 1971; Mehler, 1971; Kievit and Kuypers, 1972; Batton et al., 1977; Chan-Palay, 1977; Percheron, 1977; Stanton, 1980; Kalil, 1981; Asanuma et al, 1983b, c). Although some studies report cerebellar terminations in VLo, this conclusion probably depends on differences in the delineation of nuclear borders and has not been supported by recent experiments (see Asanuma et al., 1983a, b, c, for a complete discussion on this issue). The lack of cerebellar terminations in VLo and VLm is particularly evident in degeneration studies where large portions of the deep nuclei were removed or the brachium conjunctivum was sectioned (see Fig. 15, $A$ and $B$; Kievit and Kuypers, 1972; Percheron, 1977; but also see Stanton, 1980). A comparison of Figures 14 and 15 indicates the lack of overlap between cerebellar and pallidonigral sites of termination in the ventrolateral thalamus.

Thalamic projections from the deep nuclei appear to be topographically organized. Projections from caudal regions of each deep cerebellar nucleus terminate medially in the ventrolateral thalamus in area X. In contrast, projections from rostral regions of each nucleus terminate more laterally in the ventrolateral thalamus in VPLo (for illustration, see Fig. 15, $C$ to $F$; Stanton, 1980; Kalil, 1981; Asanuma et al., 1983b, c).

Asanuma et al. (1983a, b) have suggested that the thalamic regions which receive cerebellar inputs (i.e., VPLo, VLc, area X, and VLps) should be considered a common nucleus. They termed this nucleus the "cellsparse zone," inferred that it projected to the motor cortex, and concluded that "the cerebellum appears to influence only the primary motor area" (Asanuma et al., $1983 \mathrm{~b}$, p. 261). Furthermore, they inferred that efferents from caudal regions of the deep nuclei to area $\mathrm{X}$ influenced the face representation of the motor cortex and that efferents from rostral regions of the deep nuclei to VPLo influenced arm and leg representations in the motor cortex (see Asanuma et al., 1983c, Fig. 21). Thus, the topographic organization of the cerebellothalamic pathway was thought to reflect a somatotopic organization of the deep nuclei. This inference is supported by a physiological study which reported the following description of somatotopic organization in the deep nuclei: "a tendency for the best related 'leg neurons' to be most anterior, 'face neurons' to be posterior, and 'arm neurons' in between" (see Brooks and Thach, 1981, p. 920, for description).

We propose an alternative interpretation of cerebellothalamic topography. A comparison of our results (Figs. 


\section{Cerebellar Input}

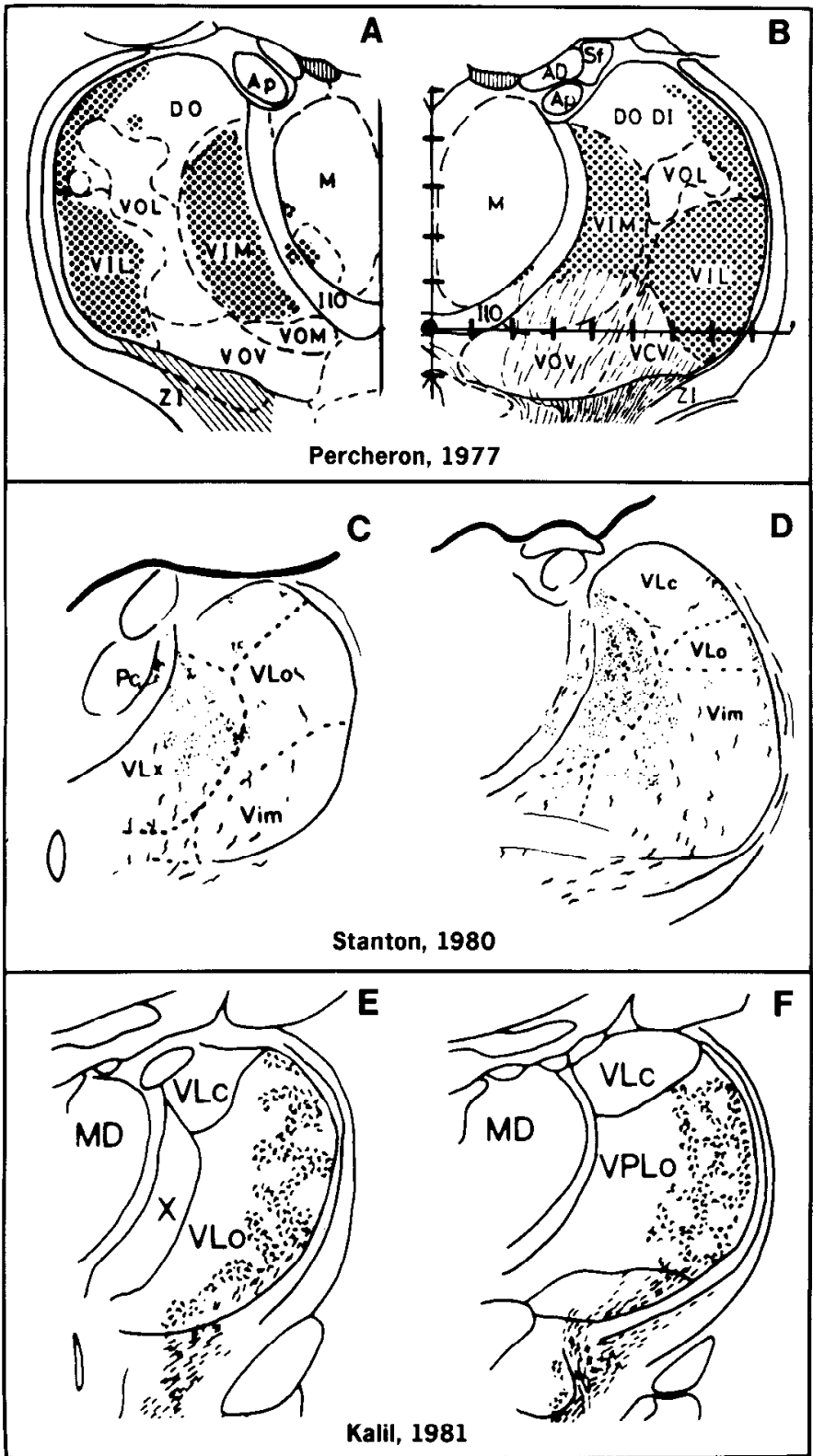

Figure 15. Pattern of cerebellothalamic termination in the ventrolateral thalamus. $A$ and $B$ are taken from the work of Percheron (1977). They represent the combined sites of termination for efferents from all three deep cerebellar nuclei at two levels in the ventrolateral thalamus. $C$ and $D$ are taken from the work of Stanton (1980). They represent the sites of termination of efferents from ventrocaudal portions of the dentate nucleus. $E$ and $F$ are taken from the work of Kalil (1981). They represent the sites of termination of efferents from rostral portions of the dentate nucleus. The levels of the sections chosen for illustration correspond to the levels of the normal and experimental material illustrated in Figures 2 to 4, $8,9,11$, and 12 . See the orignal publications for the definition of abbreviations used in this figure.

$8,9,11,12$, and $13 B$ ) with Figure 15 indicates that cerebellothalamic projections are not limited to regions of the ventrolateral thalamus which project only to the motor cortex. In particular, the present study indicates that area $\mathrm{X}$, which receives efferents from the cerebel- lum, is the origin of inputs to the APA and does not project to the "face" representation in motor cortex (see also, Roberts and Akert, 1963; Akert, 1964; Kunzle, 1976, 1978; Strick, 1976; Kievit and Kuypers, 1977; Jones et al., 1979, for relevant thalamocortical anatomy). We conclude that there are at least two separate cerebellothalamic systems which originate from the deep nuclei. Furthermore, these systems influence separate cortical areas. One system, located in rostral portions of the deep nuclei, projects largely to VPLo and therefore most directly influences the motor cortex. A second system, located in caudal portions of the deep nuclei, projects largely to area $\mathrm{X}$ and therefore most directly influences the APA. Additional support for these conclusions comes from physiological studies which report that cerebellar influences were directed to premotor as well as motor cortical regions (Sasaki et al., 1976, 1979). The possibility that either or both of these systems might be somatotopically organized, as suggested by the physiology (Brooks and Thach, 1981), remains to be examined.

\section{Summary and Speculation}

The scheme of connections which results from our conclusions is represented diagrammatically in Figure 16. Outputs from the cerebellum and basal ganglia supply three systems of subcortical afferents to the ventrolateral thalamus. These systems form parallel pathways to motor and premotor cortical areas. One parallel pathway

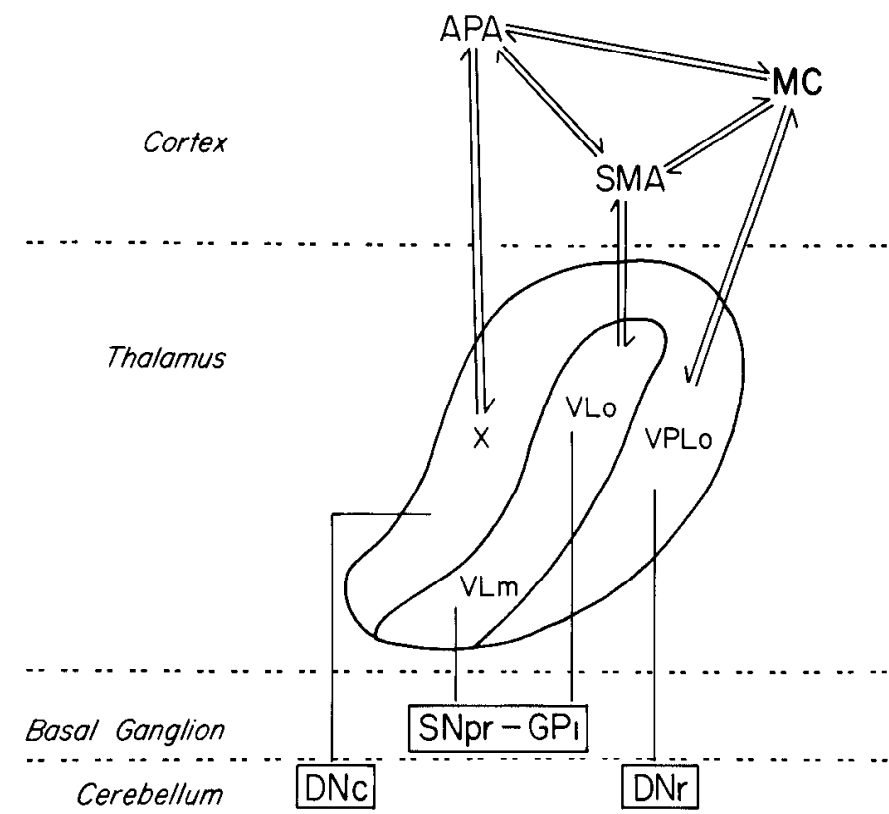

Figure 16. Summary of anatomical relationships between cerebellar and basal ganglia efferents and motor and premotor cortical areas. This diagram illustrates: (1) the pathway from caudal portions of the deep cerebellar nuclei $(D N c)$ to area $\mathrm{X}$ and the arcuate premotor area $(A P A),(2)$ the pathways from the pars reticulata of the substantia nigra $(S N p r)$ and the internal segment of the globus pallidus $(G P i)$ to VLm and VLo and the supplementary motor area $(S M A),(3)$ the pathway from rostral portions of the deep cerebellar nuclei $(D N r)$ to VPLo and the motor cortex $(M C)$, and (4) the reciprocal connections between the MC, APA, and SMA. See the text for details. 
originates in the caudal portions of the deep cerebellar nuclei and most directly influences the APA. A second pathway originates in SNpr and GPi and most directly influences the SMA. The third pathway originates in rostral portions of the deep cerebellar nuclei and most directly influences the motor cortex. Thus, each cortical area is the site of termination for a distinct subcortical system. Direct interactions between the three systems are largely limited to the cortical level where the SMA, APA, and motor cortex are reciprocally interconnected with one another. (Major interconnections between different subdivisions of the ventrolateral thalamus have not been described, although few studies have specifically examined this issue (Brodal, 1981).)

The present results suggest that the premotor areas may provide a major route by which subcortical motor nuclei influence motor cortex output. This suggestion may be particularly relevant to the basal ganglia which, unlike cerebellar nuclei, lack direct projections to thalamic regions which innervate area 4 . These results are in line with the classical view that the premotor areas serve as "links in the chain of command to motor cortex" (for discussion, see Brinkman and Porter, 1979a, b; Wiesendanger, 1981). However, one might examine the function of the premotor areas from a different perspective. The premotor areas may have functions which, in some respects, are independent of motor cortex output. Anatomical studies have shown that the SMA and cortical regions rostral to the motor cortex have direct projections to the spinal cord and to brainstem regions involved in motor control (e.g., Kuypers, 1960; Liu and Chambers, 1964; Kuypers and Lawrence, 1967; Biber et al., 1978; Murray and Coulter, 1981; for a recent review, see Kuypers, 1981). Furthermore, it is an assumption that the major direction of influence between these cortical fields is from premotor areas to the motor cortex rather than from the motor cortex to premotor areas. The results of recent studies support the concept that the SMA, APA, and motor cortex are anatomically and physiologically distinct cortical fields (e.g., Brinkman and Porter, 1979a, b; Matsumura and Kubota, 1979; Muakkassa and Strick, 1979; Roland et al., 1980a, b; Wise and Tanji, 1981; Weinrich and Wise, 1982; see also Evarts, 1981; Wiesendanger, 1981). Thus, although the SMA and APA are interconnected with the motor cortex, it may be important to view the three cortical areas as components of functionally distinct efferent systems which are driven by largely separate subcortical nuclei.

There is much to be understood about the normal function and pathophysiology of these efferent systems. The anatomical relationship between the basal ganglia and the SMA suggests that some of the movement disorders associated with basal ganglia dysfunction might be mediated by the SMA. Akinesia, which Denny-Brown (Denny-Brown, 1962; Denny-Brown and Yanagisawa, 1976) views as the primary deficit in human basal ganglia disorders, also is produced by lesions involving the SMA in humans (LaPlane et al., 1977; Damasio and Van Hoesen, 1980). It is also interesting to note the similarity between Marsden's suggestion that "the basal ganglia run a sequence of motor programs to achieve a motor plan" (Marsden, 1982) and studies that show selective increase in regional cerebral blood flow in the SMA of humans during the internal programming of motor sequences (Roland et al., 1980a).

Frontal lobe lesions in humans, in the region comparable to the APA, can result in a complex disorder of learned skilled movements termed apraxia (Geschwind, 1965; Heilman, 1979). The anatomical relationship between the cerebellum and the APA suggests the possibility of cerebellar involvement in the motor functions of the frontal lobe. Future studies might search for "apraxic-like" symptoms following lesions which involve caudal cerebellar efferent systems.

\section{References}

Akert, K. (1964) Comparative anatomy of frontal cortex and thalamofrontal connections. In The Frontal Granular Cortex and Behavior, J. M. Warren and K. Akert, eds., pp. 372-394, McGraw-Hill Book Co., New York.

Asanuma, C., W. T. Thach, and E. G. Jones (1983a) Cytoarchitectonic delineation of the ventral lateral thalamic region in the monkey. Brain Res. Rev. 5: 219-235.

Asanuma, C., W. T. Thach, and E. G. Jones (1983b) Distribution of cerebellar terminations in the ventral lateral thalamic region of the monkey. Brain Res. Rev. 5: 237-265.

Asanuma, C., W. T. Thach, and E. G. Jones (1983c) Anatomical evidence for segregated focal groupings of efferent cells and their terminal ramifications in the cerebellothalamic pathway of the monkey. Brain Res. Rev. 5: 267-297.

Batton, R. R., A. Jayaraman III, D. Ruggiero, and M. B. Carpenter (1977) Fastigial efferent projections in the monkey: An autoradiographic study. J. Comp. Neurol. 174: 281306.

Biber, M. P., L. W. Kneisley, and J. H. LaVail (1978) Cortical neurons projecting to the cervical and lumbar enlargements of the spinal cord in young and adult rhesus monkeys. Exp. Neurol. 59: 492-508.

Bowker, R. M., E. A. Murray, and J. D. Coulter (1979) Intracortical and thalamic connections of the supplementary sensory and supplementary motor areas in the monkey. Soc. Neurosci. Abstr. 5: 704.

Brinkman, J., and R. Porter (1979a) "Premotor" area of the monkey's cerebral cortex: Activity of neurons during performance of a learned motor task. Proc. Aust. Physiol. Pharmacol. Soc. 10: 198.

Brinkman, C., and R. Porter (1979b) Supplementary motor area in the monkey: Activity of neurons during performance of a learned motor task. J. Neurophysiol. 42: 681-709.

Brodal, A. (1981) Neurological Anatomy in Relation to Clinical Medicine, Oxford university Press, New York.

Brooks, V. B., and W. T. Thach (1981) Cerebellar control of posture and movement. In Handbook of Physiology. Section I: The Nervous System. Vol. II: Motor Control, V. B. Brooks, ed., pp. 877-946, American Physiological Society, Bethesda, MD.

Carmel, P. W. (1970) Efferent projections of the ventral anterior nucleus of the thalamus in the monkey. Am. J. Anat. 128: 159-184.

Carpenter, M. B., and R. E. McMasters (1964) Lesions of the substantia nigra in the rhesus monkey. Efferent fiber degeneration and behavioral observations. Am. J. Anat. 114: 293320.

Carpenter, M. B., and P. Peter (1972) Nigrostriatal and nigrothalamic fibers in the rhesus monkey. J. Comp. Neurol. 144: $93-116$.

Carpenter, M. B., and N. L. Strominger (1967) Efferent fiber projections of the subthalamic nucleus in the rhesus monkey. A comparison of the efferent projections of the subthalamic 
nucleus, substantia nigra, and globus pallidus. Am. J. Anat. 121: 41-72.

Carpenter, M. B., K. Nakano, and R. Kim (1976) Nigrothalamic projections in the monkey demonstrated by autoradiographic techniques. J. Comp. Neurol. 165: 401-416.

Carpenter, M. B., S. C. Carleton, J. T. Keller, and P. Conte (1981) Connections of the subthalamic nucleus in the monkey. Brain Res. 224: 1-29.

Chan-Palay, V. (1977) Cerebellar Dentate Nucleus. Organization, Cytology and Transmitters, Springer-Verlag, Berlin.

Damasio, A. R., and G. W. Van Hoesen (1980) Structure and function of the supplementary motor area. Neurology 30 : 359.

DeLong, M. R., and A. P. Georgopoulos (1979) Functional organization of the substantia nigra, globus pallidus, and subthalamic nucleus in the monkey. In The Extrapyramidal System and its Disorders, L. J. Poirier, T. L. Sourkes, and P. J. Bedard, eds., pp. 131-140, Raven Press, New York.

DeLong, M. R., and A. P. Georgopoulos (1981) Motor functions of the basal ganglia. In Handbook of Physiology. Section I: The Nervous System. Vol. II: Motor Control, V. B. Brooks, ed., pp. 1017-1061, American Physiological Society, Bethesda, MD.

DeLong, M. R., M. D. Crutcher, and A. P. Georgopoulos (1983) Relations between movement and single cell discharge in the substantia nigra of the behaving monkey. J. Neurosci. 3: 1599-1606.

Denny-Brown, D. (1962) The Basal Ganglia and Their Relation to Disorders of Movement, Oxford University Press, London.

Denny-Brown, D., and N. Yanagisawa (1976) The role of the basal ganglia in the initiation of movement. In The Basal Ganglia, M. D. Yahr, ed., pp. 115-148, Raven Press, New York.

DeVito, J. L., and M. E. Anderson (1982) An autoradiographic study of efferent connections of the globus pallidus in Macaca mulatta. Exp. Brain Res. 46: 107-117.

DeVito, J. L., and O. A. Smith, Jr. (1959) Projections from the mesial frontal cortex (supplementary motor area) to the cerebral hemispheres and brain stem of the Mucaca mulatta. J. Comp. Neurol. 111: 261-277.

Evarts, E. V. (1981) Role of motor cortex in voluntary movements in primates. In Handbook of Physiology. Section I: The Nervous System. Vol. II: Motor Control, V. B. Brooks, ed., pp. 1083-1120, American Physiological Society, Bethesda, MD.

Geschwind, W. (1965) Disconnexion syndromes in animals and man. Part II. Brain 88: 585-644.

Heilman, K. M. (1979) Apraxia. In Clinical Neuropsychology, K. M. Heilman and E. Valenstein, eds., pp. 159-185, Oxford University Press, New York.

Hikosaka, O., and R. H. Wurtz (1983) Visual and oculomotor functions of monkey substantia nigra pars reticulata. III. Memory-contingent visual and saccade responses. J. Neurophysiol. 49: 1268-1284.

Jones, E. G., and T. P. S. Powell (1970) An anatomical study of converging sensory pathways within the cerebral cortex of the monkey. Brain 93: 793-820.

Jones, E. G., S. P. Wise, and J. D. Coulter (1979) Differential thalamic relationships of sensory-motor and parietal cortical fields in monkeys. J. Comp. Neurol. 183: 833-882.

Kalil, K. (1978) Neuroanatomical organization of the primate motor system: Afferent and efferent connections of the ventral thalamic nuclei. In Multidisciplinary Perspectives in Event-Related Brain Potential Research, O. A. Otto, ed., pp. 112-123, U. S. Environmental Protection Agency, Washington, D. C.

Kalil, K. (1981) Projections of the cerebellar and dorsal column nuclei upon the thalamus of the rhesus monkey. J. Comp. Neurol. 195: 25-50.
Kievit, J., and H. G. J. M. Kuypers (1972) Fastigial cerebellar projections to the ventrolateral nucleus of the thalamus and the organization of the descending pathways. In Corticothalamic Projections and Sensorimotor Activities, T. Frigyesi, E. Rinvik, and M. D. Yahr, eds., pp. 91-114, Raven Press, New York.

Kievit, J., and H. G. J. M. Kuypers (1977) Oryanization of the thalamo-cortical connections to the frontal lobe in the rhesus monkey. Exp. Brain Res. 29: 299-322.

Kim, R., K. Nakano, A. Jayaraman, and M. B. Carpenter (1976) Projections of the globus pallidus and adjacent structures: An autoradiographic study in the monkey. J. Comp. Neurol. 169: 263-289.

Kubota, K., and I. Hamada (1978) Visual tracking and neuron activity in the post-arcuate area in monkeys. J. Physiol. (Paris) 74: 297-312.

Kunzle, H. (1976) Thalamic projections from the precentral motor cortex in Macaca fascicularis. Brain Res. 105: 253-267.

Kunzle, H. (1978) An autoradiographic analysis of the efferent connections from premotor and adjacent prefrontal regions (areas 6 and 9) in Macaca fascicularis. Brain Behav. Evol. 15: $185-234$.

Kuo, J-S., and M. B. Carpenter (1973) Organization of pallidothalamic projections in rhesus monkey. J. Comp. Neurol. 151: 201-236.

Kusama, T., M. Mabuchi, and T. Sumino (1971) Cerebellar projections to the thalamic nuclei in monkeys. Proc. Jpn. Acad. 47: 505-510.

Kuypers, H. G. J. M. (1960) Central cortical projections to motor and somato-sensory cell groups. An experimental study in the rhesus monkey. Brain 83: 161-184.

Kuypers, H. G. J. M. (1981) Anatomy of the descending pathways. In Handbook of Physiology. Section I: The Nervous System. Vol. II: Motor Control, V. B. Brooks, ed., pp. 597666, American Physiological Society, Bethesda, MD.

Kuypers, H. G. J. M., and D. G. Lawrence (1967) Cortical projections to the red nucleus and the brain stem in the rhesus monkey. Brain Res. 4: 151-188.

Kwan, H. C., W. A. MacKay, J. T. Murphy, and Y. C. Wong (1978) Spatial organization of precentral cortex in awake primates. II. Motor outputs. J. Neurophysiol. 41: 1120-1131.

LaPlane, D., J. Talairach, V. Meininger, J. Bancaud, and J. M. Orgogozo (1977) Clinical consequences of corticectomies involving the supplementary motor area in man. J. Neurol. Sci. 34: 301-314.

Liu, C. N., and W. W. Chambers (1964) An experimental study of the corticospinal system in the monkey (Macaca mulatta). J. Comp. Neurol. 123: 257-284.

Marsden, C. D. (1982) The mysterious motor function of the basal ganglia: The Robert Wartenberg Lecture. Neurology 32: $514-539$.

Matsumura, M., and K. Kubota (1979) Cortical projection to hand-arm motor area from post-arcuate in macaque monkeys: A histological study of retrograde transport of horseradish peroxidase. Neurosci. Lett. 11: 241-246.

Mehler, W. R. (1971) Idea of a new anatomy of the thalamus. J. Psychiatr. Res. 8: 203-217.

Mesulam, M.-M. (1978) Tetramethyl benzidine for horseradish peroxidase neurohistochemistry: A non-carcinogenic blue reaction product with superior sensitivity for visualizing neural afferents and efferents. J. Histochem. Cytochem. 26: 106117.

Mesulam, M.-M. (1982) Tracing Neural Connections with Horseradish Peroxidase, John Wiley \& Sons, Inc., New York. Mesulam, M.-M., E. Hegarty, H. Barbas, K. A. Carson, E. C. Gower, A. G. Knapp, M. B. Moss, and E. J. Mufson (1980) Additional factors influencing sensitivity in the tetramethyl benzidine method for horseradish peroxidase neurohistochemistry. J. Histochem. Cytochem. 28: 1255-1259. 
Muakkassa, K. F., and P. L. Strick (1979) Frontal lobe inputs to primate motor cortex: Evidence for four somatotopically organized "premotor" areas. Brain Res. 177: 176-182.

Murray, E. A., and J. D. Coulter (1981) Organization of corticospinal neurons in the monkey. J. Comp. Neurol. 195: 339365.

Nauta, W. J. H., and W. R. Mehler (1966) Projections of the lentiform nucleus in the monkey. Brain Res. 1: 3-42.

Olszewski, J. (1952) The Thalamus of the Macaca Mulatta. An Atlas for Use with the Stereotaxic Instrument, S. Karger AG, Basel.

Pandya, D. N., and H. G. J. M. Kuypers (1969) Cortico-cortical connections in the rhesus monkey. Brain Res. 13: 13-36.

Pandya, D. N., and L. A. Vignolo (1971) Intra- and interhemispheric projections of the precentral, premotor, and arcuate areas in the rhesus monkey. Brain Res. 26: 217-233.

Percheron, G. (1977) The thalamic territory of cerebellar afferents and the lateral region of the thalamus of the macaque in stereotaxic ventricular coordinates. J. Hirnforsch. 18: 375400.

Roberts, T. S., and K. Akert (1963) Thalamo-cortical connections and cytoarchitecture of opercular and insular cortex in Macaca mulatta. Schweiz. Arch. Neurol. Neurochir. Psychiatr. 92: 1-43.

Roland, P. E., B. Iarsen, N. A. Lassen, and E. Skinhoj (1980a) Supplementary motor area and other cortical areas in organization of voluntary movements in man. J. Neurophysiol. 43: $118-136$

Roland, P. E., E. Skinhoj, N. A. Lassen, and B. Larsen (1980b) Different cortical areas in man in organization of voluntary movements in extrapersonal space. J. Neurophysiol. 43: 137150.

Rosene, D. L., and M.-M. Mesulam (1978) Fixation variables in horseradish peroxidase neurohistochemistry. I. The effects of fixation time and perfusion procedures upon enzyme activity. J. Histochem. Cytochem. 26: 28-39.

Sasaki, K., S. Kawaguchi, H. Oka, M. Sakai, and N. Mizuno (1976) Electrophysiological studies on the cerebellocerebral projections in monkeys. Exp. Brain Res. 24: 495-507.

Sasaki, K., K. Jinnai, H. Gemba, S. Hashimoto, and N. Mizuno
(1979) Projection of the cerebellar dentate nucleus onto the frontal association cortex in monkeys. Exp. Brain Res. 37: 193-198.

Smith, A. M. (1979) The activity of supplementary motor area neurons during a maintained precision grip. Brain Res. 172: $315-327$.

Stanton, G. B. (1980) Topographical organization of ascending cerebellar projections from the dentate and interposed nuclei in Macaca mulatta: An anterograde degeneration study. J. Comp. Neurol. 190: 699-731.

Strick, P. L. (1975) Multiple sources of thalamic input to the primate motor cortex. Brain Res. 88: 372-377.

Strick, P. L. (1976) Anatomical analysis of ventrolateral thalamic input to primate motor cortex. J. Neurophysiol. 39: 1020-1031.

Tanji, J., and K. Kurata (1982) Comparison of movementrelated activity in two cortical motor areas of primates. J. Neurophysiol. 48: 633-653.

Tanji, J., K. Taniguchi, and T. Saga (1980) The supplementary motor area: Neuronal responses to motor instructions. J. Neurophysiol. 43: 60-68.

Weinrich, M., and S. P. Wise (1982) The premotor cortex of the monkey. J. Neurosci. 2: 1329-1344.

Wiesendanger, M. (1981) Organization of secondary motor areas of cerebral cortex. In Handbook of Physiology. Section I: The Nervous System. Vol. II: Motor Control, V. B. Brooks, ed., pp. 1121-1147, American Physiological Society, Bethesda, MD.

Wiesendanger, M., J. J. Seguin, and H. Kunzle (1973) The supplementary motor area. A control system for posture? Adv. Behav. Biol. 7: 331-346.

Wise, S. P., and J. Tanji (1981) Supplementary and precentral motor cortex: Contrast in responsiveness to peripheral input in the hindlimb area of the unanesthetized monkey. J. Comp. Neurol. 195: 433-451.

Woolsey, C. W., P. W. Settlage, D. R. Meyer, W. M. Spencer, T. P. Hamuy, and A. M. Travis (1952) Patterns of localization in precentral and "supplementary" motor areas and their relations to the concept of a premotor area. Res. Publ. Assoc. Res. Nerv. Ment. Dis. 30: 238-264. 